Draft Version September 12, 2013

Preprint typeset using $\mathrm{LAT}_{\mathrm{E}} \mathrm{X}$ style emulateapj v. 6/22/04

\title{
STELLAR COLLISIONS AND BLUE STRAGGLER STARS IN DENSE GLOBULAR CLUSTERS
}

Sourav ChatterJee

Department of Astronomy, University of Florida, Gainesville, FL 32611.

FREDERIC A. RASIO

Center for Interdisciplinary Exploration and Research in Astrophysics (CIERA) and Dept. of Physics \& Astronomy, Northwestern University, 2145 Sheridan Rd, Evanston, IL 60208, USA.

\author{
Alison Sills \\ Department of Physics and Astronomy, McMaster University, 1280 Main Street West, Hamilton, ON, L8S 4M1, CANADA. \\ AND \\ Evert GLeBBeEK \\ Department of Astrophysics/IMAPP, Radboud University Nijmegen, PO Box 9010, 6500 GL, Nijmegen, The Netherlands. \\ Draft version September 12, 2013
}

\begin{abstract}
Blue straggler stars (BSS) are abundantly observed in all Galactic globular clusters (GGC) where data exist. However, observations alone cannot reveal the relative importance of various formation channels or the typical formation times for this well studied population of anomalous stars. Using a state-of-the-art Hénon-type Monte Carlo code that includes all relevant physical processes, we create 128 models with properties typical of the observed GGCs. These models include realistic numbers of single and binary stars, use observationally motivated initial conditions, and span large ranges in central density, concentration, binary fraction, and mass. Their properties can be directly compared with those of observed GGCs. We can easily identify the BSSs in our models and determine their formation channels and birth times. We find that for central densities above $\sim 10^{3} M_{\odot} \mathrm{pc}^{-3}$ the dominant formation channel is stellar collisions while for lower density clusters, mass transfer in binaries provides a significant contribution (up to $60 \%$ in our models). The majority of these collisions are binary-mediated, occurring during 3-body and 4-body interactions. As a result a strong correlation between the specific frequency of BSSs and the binary fraction in a cluster can be seen in our models. We find that the number of BSSs in the core shows only a weak correlation with the collision rate estimator $\Gamma$ traditionally used by observers, in agreement with the latest Hubble Space Telescope (ACS) data. Using an idealized "full mixing" prescription for collision products, our models indicate that the BSSs observed today may have formed several Gyrs ago. However, denser clusters tend to have younger $(\sim 1 \mathrm{Gyr})$ BSSs.

Subject headings: methods: numerical — methods: statistical — blue stragglers — stars: kinematics and dynamics - globular clusters: general
\end{abstract}

\section{INTRODUCTION}

Blue straggler stars (BSSs) are H-burning stars bluer and brighter (and therefore more massive) than the mainsequence turnoff (of a star cluster; Sandage 1953). It is impossible for normal single star evolution to create them. Instead, BSSs must be created when a mainsequence (MS) star's mass is increased either via mass transfer in a binary (MTB) or by physical collision and merger with another star. Both processes can also increase the residual H-burning life of the star (rejuvenation) by bringing in new Hydrogen for burning in the core (eg., Lombardi et al. 1995, 1996; Sills et al. 1997, 2001; Lombardi et al. 2002; Chen \& Han 2009) ${ }^{1}$. Since the BSSs are among the brighter members of a cluster, they are relatively easier to detect in photometric observations. All Galactic globular clusters (GGCs), where

\footnotetext{
Electronic address: s.chatterjee@astro.ufl.edu

${ }^{1}$ Note that even if no new Hydrogen is supplied to the core, the star may still appear as a BSS during its own residual MS life if its present mass is higher than the MS turn-off.
}

appropriate data exist, have shown this exotic stellar population (e.g., Piotto et al. 2002; Ferraro et al. 1995). Many open clusters also show moderately large populations ( 10s) of BSSs (e.g., Mathieu \& Geller 2009); they are also found in some dwarf galaxies (e.g., Mapelli et al. 2007) and even in the field (e.g., Preston \& Sneden 2000).

Stellar collisions can happen in many ways in a star cluster. Two single stars can collide directly (s-s collision). If binaries are present, then binaries will interact with other single stars (b-s) or binaries (b-b). During b-s and b-b encounters, physical collisions can occur between two or more MS stars taking part in the interactions (Fregeau et al. 2004).

During MTB the details of the orbital evolution and mass transfer rate determine the final outcomes. Mass transfer can be stable, such that a fraction of the companion mass is transferred to the MS star and the binary remains intact; or it can be unstable, leading to a more rapid dynamical evolution and the eventual merger of the two stars (Chen \& Han 2009). Stellar mergers may also happen through interactions involving higher-order 
hierarchical systems. For example, a hierarchical triple, for certain configurations can increase the eccentricity of the inner orbit via the Kozai effect (Kozai 1962). If the eccentricity becomes sufficiently high, the two inner stars could then be driven to merge (Perets \& Fabrycky 2009). Although this may be a significant channel for BSS formation in less dense open clusters (Perets \& Fabryckv 2009), it is not clear whether these triples can survive long enough in denser GGCs to be effective.

The interactions that produce BSSs depend on the cluster global properties, including the central density $\left(\rho_{c}\right)$, binary fraction $\left(f_{b}\right)$, distribution of initial binary orbital properties, and mass-segregation timescale. Thus the BSSs in a cluster can, in principle, provide a window onto the system's dynamical history. In this respect the formation channel(s) of the BSSs and their typical formation times are of great interest. Unfortunately, although observations in various wavelength bands have taught us a lot about the stellar properties of the BSSs, it is hard to determine how a particular BSS formed or how long ago the interaction occurred. Theoretical modeling is the only way to approach these questions.

A popular approach so far to learn about BSS formation has been to carefully study the correlation between the number of BSSs (normalized in some way by another characteristic stellar population in the same region of the cluster) and some dynamically important cluster property, such as the collision rate estimator $\Gamma$ (Pooley \& Hut 2006), $f_{b}$ (Sollima et al. 2008), or the total cluster mass (most recently Leigh et al. 2013). While these observed correlations can in principle teach us a lot about the possible formation channels of BSSs, direct understanding of these processes is impossible to achieve without careful modeling. For example, a BSS created via MTB could actually be the result of some past dynamical encounter that changed the binary orbit in such a way that mass transfer occurred. On the other hand, a collision may be mediated by resonant dynamical interactions involving binaries, blurring the distinction between the simple "collisional" and "binary" origins often discussed in observational studies (e.g., Knigge et al. 2009; Leigh et al. 2011a). In addition to these complications, it is also hard to determine observationally what the binary fraction in the cluster is at present, and it is impossible to know what it was in the past. Even in the complete absence of binaries, the calculation of the $\Gamma$ parameter involves many properties of the cluster that are hard to measure accurately.

The only way to explore and probe these complicated interactions in an evolving cluster is by numerical modeling of the full $N$-body system with stellar evolution, using a realistically large $N$, the full stellar mass function, and treating binary stars in detail. One must then also include all relevant physical processes, such as twobody relaxation, strong encounters including physical collisions, b-b and b-s interactions, MTB, tidal stripping in the Galactic potential, etc. In these models, if all interactions are recorded, in addition to the overall cluster properties and individual stellar properties at frequent enough output times, then all necessary information can be obtained about BSS formation and evolution. In particular, after BSSs have been identified in any snapshot of the system, the detailed past history of each BSS going all the way back to $t=0$ can be extracted from the numerical data.

For many years, this kind of realistic modeling of GGCs (particularly using high enough $N$ and $f_{b}$ ) remained very challenging because of the extreme computational cost of direct $N$-body simulations. Hence, much more simplified models were used to study the BSS formation channels and other properties such as their radial distribution in the cluster (Mapelli et al. 2006). Recently, modern Hénon-type Monte Carlo codes (Hénon 1971), modified to include the relevant physical processes (in particular, strong interactions and single and binary stellar evolution), have opened new possibilities (e.g., Chatteriee et al. 2010; Giersz \& Heggie 2011; Hvpki \& Giersz 2013; Chatteriee et al. 2013; Giersz et al. 2013).

The current version of our Cluster Monte Carlo code (CMC) is well suited to the modeling of GGCs including all relevant physical processes, with large $N \sim 10^{5}-10^{6}$ and realistic $f_{b}$ values. $\mathrm{CMC}$ has been developed and rigorously tested for over a decade (Joshi et al. 2000, 2001; Fregeau et al. 2003; Fregeau \& Rasio 2007; Chatteriee et al. 2010; Umbreit et al. 2012; Pattabiraman et al. 2013; Chatterjee et al. 2013). Using this code it is now possible to produce large numbers of highly detailed cluster models covering the full range of observationally motivated initial parameters. For example, recent studies with $\mathrm{CMC}$ have explored a large range in parameter space and successfully created a library of cluster models that have properties very similar to the observed GGCs (Chatterjee et al. 2010, 2013).

Here we report results from our analysis of 128 cluster models produced with $\mathrm{CMC}$ with finely sampled output focusing on BSS formation and evolution. These models include all relevant physical processes, and include realistic $N$, and $f_{b}$, and hence can be directly compared with observed GGCs without any need for rescaling. In addition to all dynamical properties, single and binary stellar evolution is also followed in detail in these models. Thus our simulations provide an unprecedented opportunity to understand the dominant dynamical and stellar evolution processes responsible for BSS production in clusters, spanning a large range in cluster parameters, and allow us to test for various correlations between BSSs and cluster properties.

Previous works have pointed out that some dynamical properties such as core radius $\left(r_{c}\right)$ or $\rho_{c}$ for a cluster can have very different values according to different definitions used by theorists and observers (most recently Chatterjee et al. 2013). Hence, to avoid confusion, we restrict our analysis in this paper to the standard theoretical definitions used in most $N$-body simulations (Casertano \& Hut 1985), unless otherwise specified. A different and independent analysis of the same models is presented in Sills et al. (2013), where all relevant quantities are determined using the standard observational definitions. In this paper we focus on two basic questions: (1) What is the dominant physical process that creates the BSSs observed in GGCs? (2) With our adopted rejuvenation prescription (Hurlev et al. 2000, 2002), what is the typical age $\left(t_{\text {age }}\right)$ of the observed BSSs?

The paper is organized as follows. In Section 2 we describe briefly our simulation setup, explain how the BSSs are identified in the models, and provide definitions 
for various categories of BSSs based on their formation histories. We also show comparisons between modeled and observed BSSs in terms of observable properties. We discuss the relative importance of the various formation channels in Section 3. The time elapsed since formation for the BSSs in our models is explored in Section 4 In Section 5 we investigate correlations of the number of BSSs in the cluster $\left(N_{\mathrm{BSS}}\right)$ with $f_{b}$ and $\Gamma$. We summarize and conclude in Section 6 .

\section{NUMERICAL METHOD}

We use CMC and adopt a large grid of initial conditions over the range of values typical of the observed young massive clusters (e.g., Scheepmaker et al. 2007, 2009) to create 128 detailed star-by-star models. All our simulated clusters have initial virial radii between $r_{v}=3$ and 4 pc. Initial $N$ is varied between 4 and $10 \times 10^{5}$ stars. The positions and velocities of the stars (and center of mass for the binaries) are assigned according to King profiles with $W_{0}$ between 4 and 8 . We vary the initial $f_{b}$ between 0.05 and 0.3 . The masses of the single stars, or primaries in case of a binary, are chosen from the IMF presented in Kroupa (2001, their Equations 1 and 2) in the stellar mass range $0.1-100 \mathrm{M}_{\odot}$. Secondary binary companion masses are sampled from a uniform distribution of mass ratios in the range $0.1 \mathrm{M}_{\odot}-\mathrm{m}_{\mathrm{p}}$, where $m_{p}$ is the mass of the primary. The semi-major axes, $a$, for stellar binaries are chosen from a distribution flat in $\log a$ within physical limits, namely between $5 \times$ the physical contact of the components and the local hard-soft boundary (Heggie \& Hut 2003). Although initially all binaries in our models are hard at their respective positions, some of these hard binaries can become soft during the evolution of the cluster. The cluster contracts under two-body relaxation and the velocity dispersion increases making initially hard binaries soft. Moreover, binaries sink to the core due to mass segregation where the velocity dispersion is higher than that at the initial binary positions. We include these soft binaries in our simulations until they are naturally disrupted via strong encounters in the cluster.

The numerical setup and initial properties for these simulations are similar to the work presented in Chatteriee et al. (2013) which shows that similar initial conditions result in models at the end of the simulation (at cluster age $t_{\mathrm{cl}} \approx 12 \mathrm{Gyr}$ ) with properties including $r_{c}$, half-mass radius $\left(r_{h}\right), \rho_{c}$, total mass $(M)$, and relaxation timescale at half-mass $\left(t_{r h}\right)$ very similar to those observed in the GGCs. The differences in the initial conditions between these simulations and those presented in Chatterjee et al. (2013) are as follows. Models with zero initial $f_{b}$ are discarded in this study since observations suggest that this is not the case for the GGCs in reality (Davis et al. 2008; Milone et al. 2012) ${ }^{2}$. In addition, the models presented in this study explore a larger range in initial $f_{b}$, and $W_{0}$.

While in most cases we present theoretical estimates of central quantities such as $r_{c}$ and $\rho_{c}$ Casertano \& Hut 1985), whenever we directly compare model quantities with observed GGC quantities, we use the corresponding

2 In fact, comparison between the observed BSS numbers in the GGCs and the numbers obtained via mock observations of these models indicates that even initial $f_{b}=0.05$ may be too low (Sills et al. 2013). "mock observed" values $\left(r_{c . \text { obs }}\right.$, and $\left.\rho_{c \text {.obs }}\right)$ to remain consistent. Most recently Chatteriee et al. (2013) discussed in detail various definitions for the cluster parameters and the relationships between theoretical and observed estimates of these quantities. Here, we estimate $r_{c, \text { obs }}$ as the radius where the surface luminosity density drops by a factor of 2 from the central density (e.g., Spitzer 1987). The values for $\rho_{c, \text { obs }}$ are estimated from the peak central surface luminosity density and $r_{c, \text { obs }}$ using the prescription given in Diorgovski (1993, their Equation 4) following Harris (1996, 2010 edition). We assume $M / L=2$ to convert luminosity to mass when required to determine the mock observed values.

Our grid of simulations in this study covers star clusters at $t_{\mathrm{cl}} \approx 12 \mathrm{Gyr}$ with $r_{c}$ between 0.6 and $2.8 \mathrm{pc}$. This range in $r_{c}$ corresponds to $r_{c, \text { obs }}$ in the range between $\approx 0.06$ and $5.07 \mathrm{pc}$. Similarly, this grid spans clusters with $\rho_{c}$ between $\approx 7 \times 10^{2}$ and $10^{5} M_{\odot} \mathrm{pc}^{-3}$ which corresponds to $\rho_{c, \text { obs }}$ between $\approx 3 \times 10^{2}$ and $4 \times 10^{6} M_{\odot} \mathrm{pc}^{-3}$. Final $M$ are between $1 \times 10^{5}$ and $4 \times 10^{5} M_{\odot}$, and corresponding $M_{\text {obs }}$ values are similar. Among the 128 models, 19 have reached the binary-burning stage (thought to be equivalent to the post core-collapsed GGCs) before $t_{\mathrm{cl}}=12 \mathrm{Gyr}$, while the rest are still contracting (i.e., they are non-core-collapsed GGCs, Chatteriee et al. 2013). Relevant model properties are listed in Table 1. For more detailed comparison between model and GGC properties see Chatteriee et al. (2013). For mock observed BSS values for these models and further comparisons with GGC properties see Sills et al. (2013).

\subsection{Definition of blue straggler stars in our models}

We define BSSs as stars that are still in the H-burning stage but with masses $>1.1 M_{\mathrm{TO}}$, where, $M_{\mathrm{TO}}$ is the MS turn-off mass of the cluster. Note that in real clusters BSSs are chosen from a color-magnitude diagram by a defining box for the BSSs that depend on, for example, the quality of the data, the filters used for the observation, and the scatter width of the MS (e.g., Leigh et al. 2007; Sills et al. 2013). The mass-based criterion is more theoretically motivated and is much simpler to use for all models (although, inaccessible to observers). We find that in our models the BSSs identified using the massbased criterion populate the expected region of synthetic Hertzsprung-Russell diagram (HRD) created using our models (Figure 1 for an example). Observationally motivated selection boxes are adopted to extract BSSs from these same models in Sills et al. (2013) and we find that the numbers obtained from either criteria are tightly correlated. Furthermore, the selected BSSs using either criteria do not show any systematic differences in their formation channels, formation ages, or radial positions. Since in this study we restrict ourselves to the theoretical understanding of the BSSs, in particular to understand the dominant formation channels and typical ages since formation, we simply adopt the much simpler theoretically motivated mass-based definition for BSSs for this study.

\subsection{Classification of blue-straggler stars based on their formation channels}

At the last snapshot of the model $\left(t_{\mathrm{cl}} \sim 12 \mathrm{Gyr}\right)$ we identify the BSSs following the criterion described in Section 2.1. The full dynamical history for each of these 


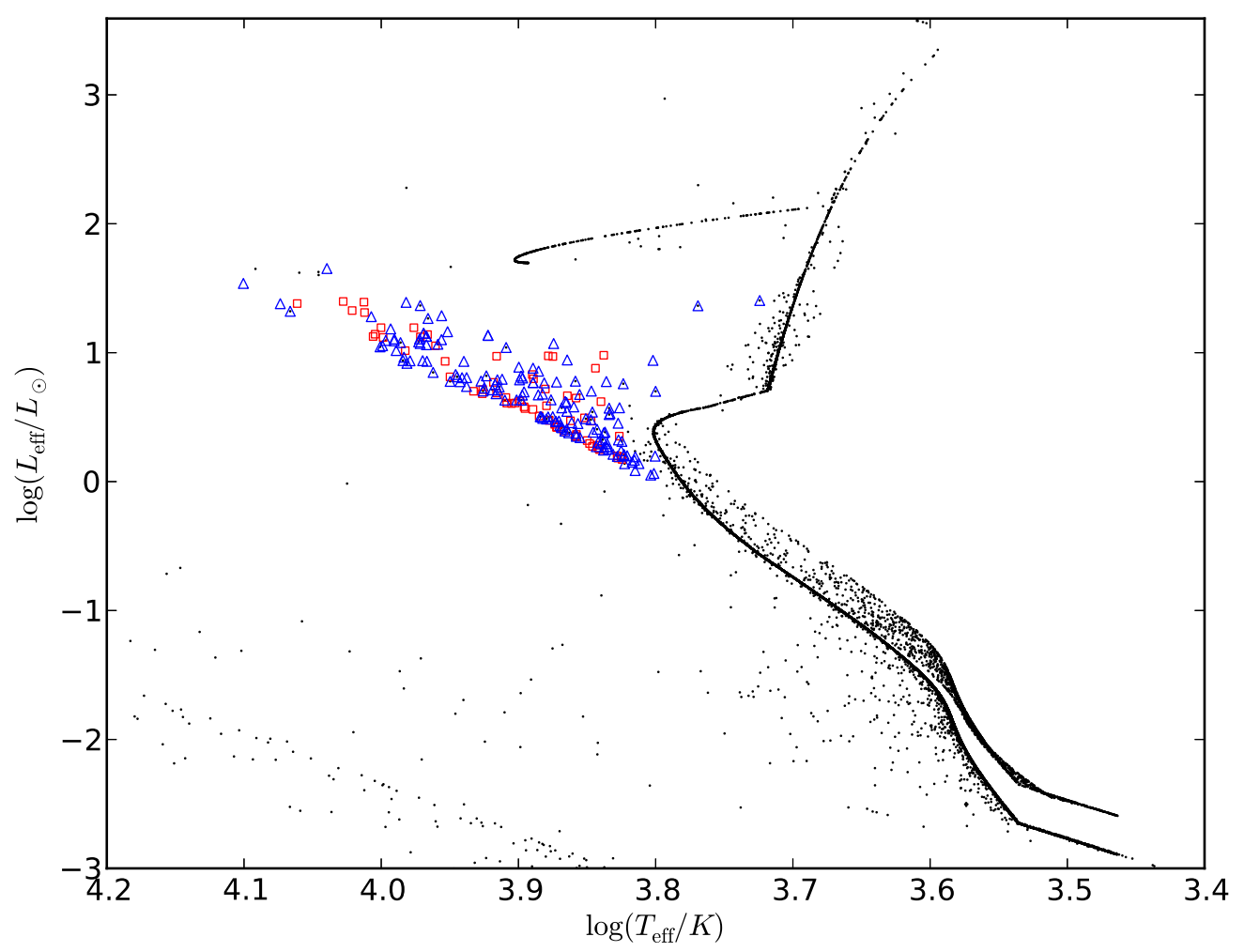

FIG. 1.- Example of a synthetic Hertzsprung-Russell (HRD) diagram for a representative model with many blue straggler stars (run110, Table 11. Dots denote all stars in the cluster. Red squares and blue triangles denote blue-straggler stars in single and binary systems, respectively at the integration stopping time of $t_{\mathrm{cl}}=12 \mathrm{Gyr}$. Blue straggler stars are defined as H-burning stars with stellar masses $\geq 1.1 M_{\mathrm{TO}}$, where $M_{\mathrm{TO}}$ is the main-sequence turn-off mass. BSSs in this model populate the expected region in the synthetic HRD. In addition, the single and binary main-sequences, giant branch, white-dwarf cooling sequence are clearly visible.

BSSs is retrieved. We identify the interaction (such as $\mathrm{s}-\mathrm{s}$, b-s, or b-b collisions, or MTB) that had increased the mass of this star for the first time such that it would be identified as a BSS at $t_{\mathrm{cl}}$. We classify each BSS into one of 5 channels according to this first interaction. The 5 channels are: stable mass transfer in a binary denoted as MTB, binary-stellar-evolution-driven merger denoted as SE, single-single collisions denoted as $\mathbf{s}-\mathbf{s}$, collisions mediated by binary-single interactions denoted as b-s, and collisions mediated by binary-binary interactions denoted as b-b. Note that some BSSs may have a complicated history. For example, after a mass transfer episode in a binary, a MS star may have attained a BSS mass; later on this star could still collide with another MS star, further growing in mass and getting rejuvenated for a second time. For BSSs that are formed via such multiple channels, only the first one is considered for the classification to avoid ambiguity. In this particular example the formation channel for this BSS would be classified as MTB. Nevertheless, such complicated multiple episodes of rejuvenation of the same MS star is not frequent. There is another source of complication. A BSS classified as MTB may have had interacted dynamically prior to the Rochelobe overflow that had not changed its mass. Such an interaction would change the orbital properties of the binary, sending it along a very different evolutionary path- way that led to the mass transfer event that ultimately created the BSS. These perturbed MTB or SE channels are duly noted and discussed. However, interactions of this type do not change the formation classification.

\subsection{Comparison of Model Blue Stragglers with Observed Blue Straggler Properties}

Here we briefly investigate whether the modeled BSS properties are consistent with some of the well studied observed BSS properties in the GGCs. Note that these are motivated to add further validation of the model BSSs. More detailed analysis of these properties are potentially interesting but beyond the scope of this study and we differ that to Sills et al. (2013) and future work.

\subsubsection{Radial Distribution}

One of the observationally accessible properties of the BSSs in the GGCs is their radial distribution. The radial distribution of the BSSs in a cluster depends on a collection of the host cluster's properties including $\rho_{c}$, relaxation time, and $f_{b}$. Thus analysis of the radial distribution of the BSSs has been proposed as a tool to observationally constrain the dominant formation channel, and various dynamical properties of the host GGCs. As a result, the radial distribution of the BSSs has 
been studied with interest for many GGCs via observations (e.g., Fusi Pecci et al. 1992; Dalessandro et al. 2008b; $\quad$ Ferraro et al. 1995; Beccari et al. 2008; Dalessandro et al. 2008a; Moretti et al. 2008al,b; Ferraro et al. 2012) as well as via theoretical modeling (e.g., Mapelli et al. 2006). Three qualitatively different types of radial distributions are observed among the GGCs, namely, single and bimodal, and flat distributions (for a compilation see Ferraro et al. 2012). We check whether the radial distributions of our model BSSs are consistent with those observed in the GGCs.

To find the relative frequency of the BSSs in the cluster as a function of their radial positions we define a double-normalized ratio $R_{\mathrm{BSS}}$ following Ferraro et al. (2012) given by

$$
R_{\mathrm{BSS}}=\frac{\delta N_{\mathrm{BSS}} / N_{\mathrm{BSS}}}{\delta L / L_{\mathrm{cl}}},
$$

where $\delta N_{\text {BSS }}$ denotes the number of BSSs within some radial bin, $N_{\text {BSS }}$ denotes the total number of BSSs, $\delta L$ denotes the stellar luminosity within that bin, and $L_{\mathrm{cl}}$ denotes the total luminosity of the cluster. $R_{\mathrm{BSS}}$ is a measure of whether the BSSs follow the luminosity density profile of the cluster or they are over/under abundant in some parts.

Figure 2 shows $R_{\mathrm{BSS}}$ as a function of the radial position in units of the core radius. In our models we find examples of bimodal distributions (bottom panel, observed, e.g., in M55) as well as centrally peaked distributions (top panel, observed, e.g., in M80, Ferraro et al. 2012, and references therein). In our models we do not find flat radial distributions observed, for example, in NGC 2419 (Dalessandro et al. 2008b). This is expected since all our models are relaxed and hence a non-segregated radial distribution for the BSSs is not expected in our models. Ferraro et al. (2012) suggest that the minimum distance $r_{\min }$ from the center where the central peak of $R_{\mathrm{BSS}}$ ends in units of $r_{c}$ is anti-correlated with the core relaxation timescale due to mass-segregation effects of the relatively high-mass BSSs. Results from our models are consistent with this finding. However, we find that the determination of $r_{\min }$ can have large errors and depends strongly on the radial bins used to calculate $R_{\mathrm{BSS}}$.

\subsubsection{Core Blue Straggler Number and Core Radius}

Are the number of BSSs in the core $\left(N_{\mathrm{BSS}, \mathrm{c}}\right)$ in our models consistent with those in the GGCs? Figure 3 shows $N_{\mathrm{BSS}, \mathrm{c}}$ as a function of $r_{c, \text { obs }}$ both for our models and for observed GGCs. Note that since we are directly comparing with observed BSSs, we use $r_{c, \text { obs }}$ for this comparison. The BSSs are, however, chosen using the same mass-based criteria. The observed BSS counts are obtained from the ACS survey data (Sarajedini et al. 2007) as selected by Leigh et al. (2011b). Our models clearly have very similar core radii compared to the observed GGCs. We identify a slightly larger $N_{\mathrm{BSS}, \mathrm{c}}$. More observationally motivated selection criteria presented in Sills et al. (2013) for the same models show that if these systems were observed, the number of BSSs actually detected would have been smaller by about a factor of 2 . Thus we conclude that $N_{\mathrm{BSS}, \mathrm{c}}$ as produced in our models and the core radii both are consistent with the observed Milky Way populations.

In addition to these caveats about different identification criteria for BSSs, one should also note that, within $\mathrm{BSE}$, and the current version of $\mathrm{CMC}$, the rejuvenation prescription used for all mergers, whether from collisions or binary evolution, and MTB assumes "full mixing" (for details see Hurlev et al. 2000, 2002). This is an oversimplification. In reality, the amount of Hydrogen core mixing and thus the degree of rejuvenation of the MS star depends on the kinematic details of the encounter (e.g., Sills et al. 1997, 2001). Because of this simplification the lifetimes of the BSSs after rejuvenation in our models are actually upper limits (at least for non-rotating stars). This may also lead to an over-production of BSSs in our models compared to real clusters.

\section{RELATIVE IMPORTANCE OF BLUE STRAGGLER FORMATION CHANNELS}

In this section we investigate the relative contributions from various BSS formation channels. Figure 4 shows an example of branching ratios for the BSSs in the whole cluster, and in the core, for model run32 (Table 11). This is a model with moderate central density $\rho_{c}=7 \times 10^{3} \mathrm{M}_{\odot} \mathrm{pc}^{-3}$. Collisions produce most of the BSSs in this model. Among the collisional BSSs, the $\mathbf{s}^{-\mathbf{s}}$ channel contributes the least. The binary mediated collisional channels, namely $\mathrm{b}-\mathrm{s}$ and $\mathrm{b}-\mathrm{b}$ contribute about $36 \%$ and $50 \%$, respectively. BSSs produced via the MTB channel contributes about $11 \%$ and SE contributes only $3.2 \%$. As expected collisional channels dominate even more among the core BSSs. Interestingly, although the $\mathrm{b}-\mathrm{s}$ and $\mathrm{b}-\mathrm{b}$ contributions increase if only core BSSs are considered instead of all BSSs in the cluster, $\mathbf{s}^{-\mathbf{s}}$ contribution decreases. This trend is seen often in our models because of the low number density of single MS stars within the core at late times, due to mass segregation.

About $60 \%$ of all BSSs formed via MTB or SE in this example model have had at least one prior strong encounter. Thus for this particular model a significant fraction of even the MTB and SE BSSs are not created via pure binary stellar evolution, and have been dynamically modified before binary stellar evolution could rejuvenate them.

Branching ratios for various BSS production channels are expected to depend on $\rho_{c}$ and $f_{b}$ in a cluster. We divide our full set of models into subsets based on the initial $f_{b}$ and present the branching ratios for each subset separately as a function of $\rho_{c}$.

Figure 5 shows the branching ratios for $\mathrm{s}-\mathrm{s}, \mathrm{b}-\mathrm{s}, \mathrm{b}-\mathrm{b}$, MTB, and SE formation channels as a function of $\rho_{c}$. The different panels show subsets of models with different initial $f_{b}$. For models with $\rho_{c}>$ few $\times 10^{3} \mathrm{M}_{\odot} \mathrm{pc}^{-3}$ collisional channels clearly dominate. In lower density clusters contribution from MTB increases. Throughout the full range in $f_{b}$ in our models $\left(f_{b}=0.05-0.30\right)$, the same trend is observed. This indicates, that even if $f_{b}$ is increased (within the studied range in $\rho_{c}$ ) the relative contribution from MTB does not increase proportionally. While increasing $f_{b}$ increases the probability that a BSS can be created via MTB (simply because of the increased number of binaries), the increased number of binaries also increases the $\mathrm{b}-\mathrm{s}$ and $\mathrm{b}-\mathrm{b}$ BSS formation rates. Typical $\rho_{c}$ values in the GGCs are orders of magnitude higher 

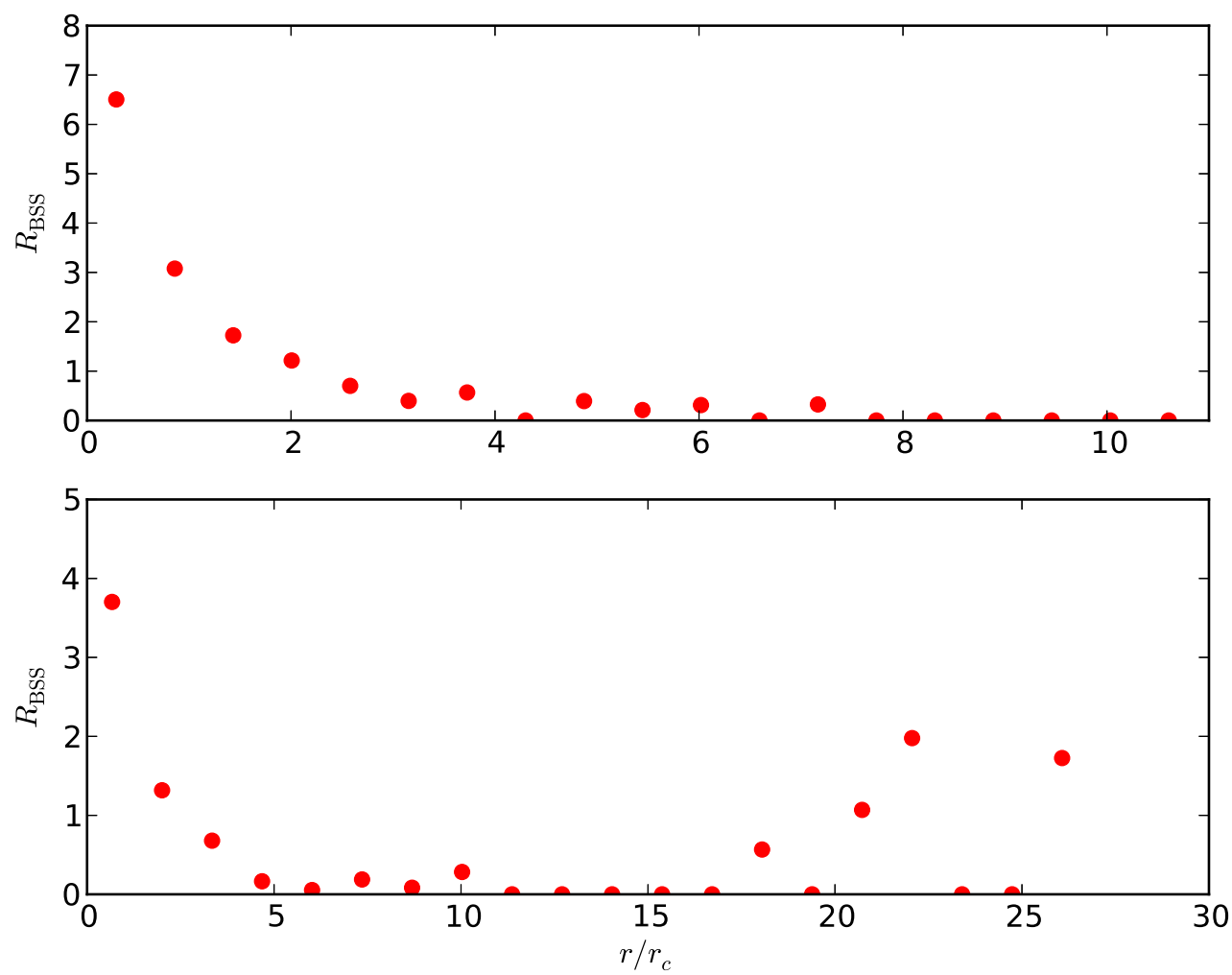

Fig. 2. - Examples of two qualitatively different radial distributions for our model BSSs. Vertical and horizontal axes denote the doublenormalized ratio $R_{\mathrm{BSS}}$ (Equation 1) and radial position in units of $r_{c}$, respectively. Top panel shows an example of centrally peaked radial distribution (run108; Table 1). Bottom panel shows an example of a bimodal distribution (run110), one peak near the center $r / r_{c}<5$ and a second peak at large $r / r_{c} \approx 22$. A zone of avoidance is observed at $r / r_{c}$ between about 5 and 17 .

than $\sim 10^{3} \mathrm{M}_{\odot} \mathrm{pc}^{-3}$ (e.g., Harris 1996; Chatteriee et al. 2010, 2013). Hence, we conclude that the collisional channels dominate production for the BSS populations observed in the majority of the GGCs. If only the core BSSs are considered, the importance of the collisional channels increases as expected. We find that throughout the full range of $\rho_{c}$ in our models collisional channels dominate the formation of core BSSs (Figure 6).

Although binary stellar evolution is not the dominant channel for a large range of central densities typical of GGCs, binaries themselves are nevertheless crucial for significant BSS production. Figure 7 and 8 show the relative contributions from various collisional formation channels for the BSSs in the whole cluster and in the core, respectively, as a function of $\rho_{c}$. For the full range in $f_{b}$ and $\rho_{c}$ in our models BSSs produced via $s^{-s}$ are rare. Binary-mediated collisions $(b-s$ or $b-b)$ dominate. As $f_{b}$ increases, so does the relative contribution from $\mathrm{b}-\mathrm{b}$. For example, for models with initial $f_{b}=0.05$, the $\mathrm{b}-\mathrm{s}$ channel contributes more to the collisional BSSs compared to the $\mathrm{b}-\mathrm{b}$ channel for all $\rho_{c}$ values. In contrast, for initial $f_{b}=0.3$ the contribution from the $\mathrm{b}-\mathrm{b}$ channel dominates for all $\rho_{c}$ values we have considered.

Figure 9 shows the fraction of BSSs produced via binary stellar evolution (MTB and SE collectively) that have been dynamically perturbed before the mass trans- fer process has occurred (with respect to all BSSs produced via MTB and SE). These interactions can potentially alter the orbital properties of these binaries initiating very different evolutionary pathways for them. BSSs formed from these disturbed binaries clearly would not have formed the BSSs with the exact same properties and at the same times without these encounters. As expected, for the models with higher $\rho_{c}$ values MTB and SE BSSs have a higher chance of being perturbed before production. For a given $\rho_{c}$ a range of these values are also seen. This is due to the different concentrations for these clusters. Models with initially lower concentrations produce a smaller fraction of perturbed MTB and SE BSSs. The majority of the models with $\rho_{c} \gtrsim 10^{4} M_{\odot} \mathrm{pc}^{-3}$ produce more perturbed MTB and SE BSSs than unperturbed. Low density $\left(\rho_{c} \lesssim 10^{3}\right)$ models on the other hand will have a relatively larger fraction of BSSs via the MTB and SE channels created from the primordial undisturbed binaries. Similar results were also found previously by Sollima et al. (2008). Our results support their conclusion that for $\rho_{c} \lesssim 10^{3} M_{\odot} \mathrm{pc}^{-3}$ stellar evolution channels contribute $\gtrsim 50 \%$ of the BSSs, and a high fraction of them are produced from dynamically unmodified binaries.

\section{TIME SINCE FORMATION FOR THE BLUE STRAGGLER STARS}




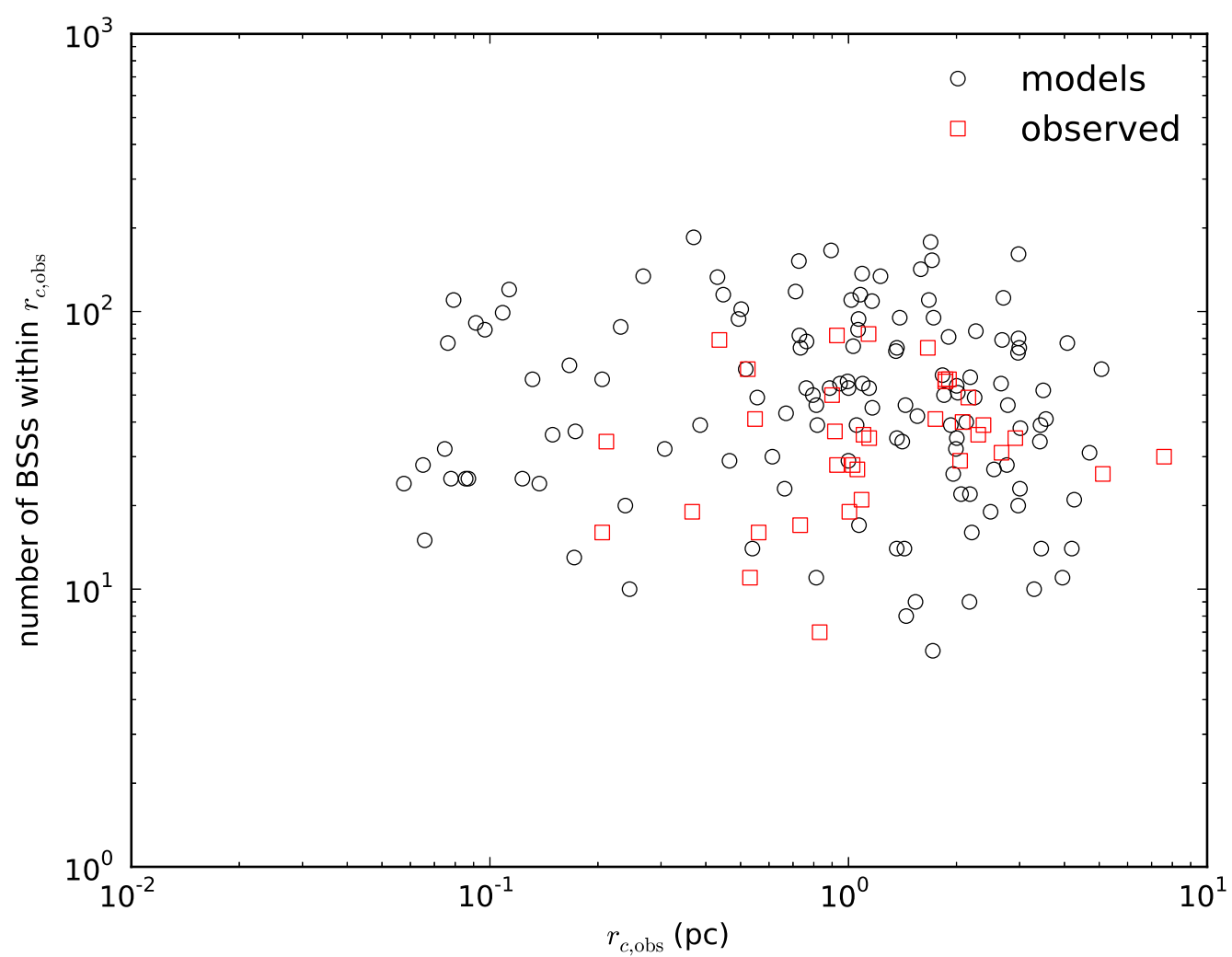

FIG. 3.- Number of BSSs in the core $\left(N_{\mathrm{BSS}, \mathrm{c}}\right)$ vs $r_{c, \text { obs }}$. Black circles and red squares denote model and observed BSSs, respectively. The observed $N_{\text {BSS,c }}$ values are obtained from the HST/ACS survey (Saraiedini et al. 2007) as selected by Leigh et al. (2011b). Our models populate very similar $r_{c, \text { obs }}$ values compared to the observed GGCs. However, our models show slightly higher $N_{B S S, c}$ numbers due to the mass-based selection criteria. If these models could be observed using the HST/ACS filters and the BSSs were identified using a more observationally motivated selection box $N_{\mathrm{BSS}, \mathrm{c}}$ would decrease by about a factor of 2 (Sills et al. 2013).

all BSSs

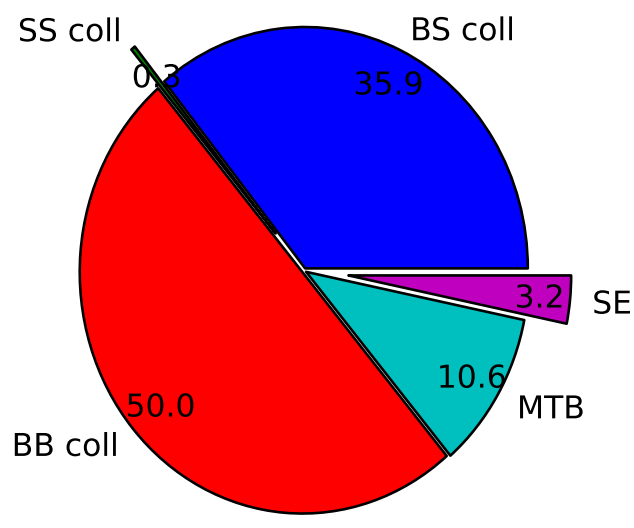

core BSSs

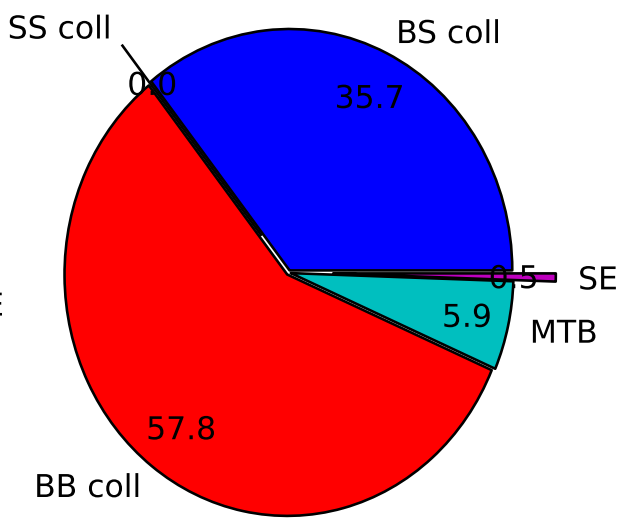

FIG. 4. - Example of branching ratios for the various formation channels of BSSs at $t_{\mathrm{cl}}=12$ Gyr (run32; Table 1). This model has a moderate central density $\left(\rho_{c} \approx 7 \times 10^{3} M_{\odot} \mathrm{pc}^{-3}\right.$; Table 1). This particular model was chosen as an example due to its high number (312) of BSSs. The dominant formation channel is stellar collisions in this model. Among the collisional BSS production channels collisions mediated by b-b interactions dominate followed by b-s mediated collisions. $\mathrm{s}-\mathrm{s}$ collisions are rare. MTB is the dominant channel among the binary-stellar-evolution driven BSS production channels. SE contribute only to a small degree. 


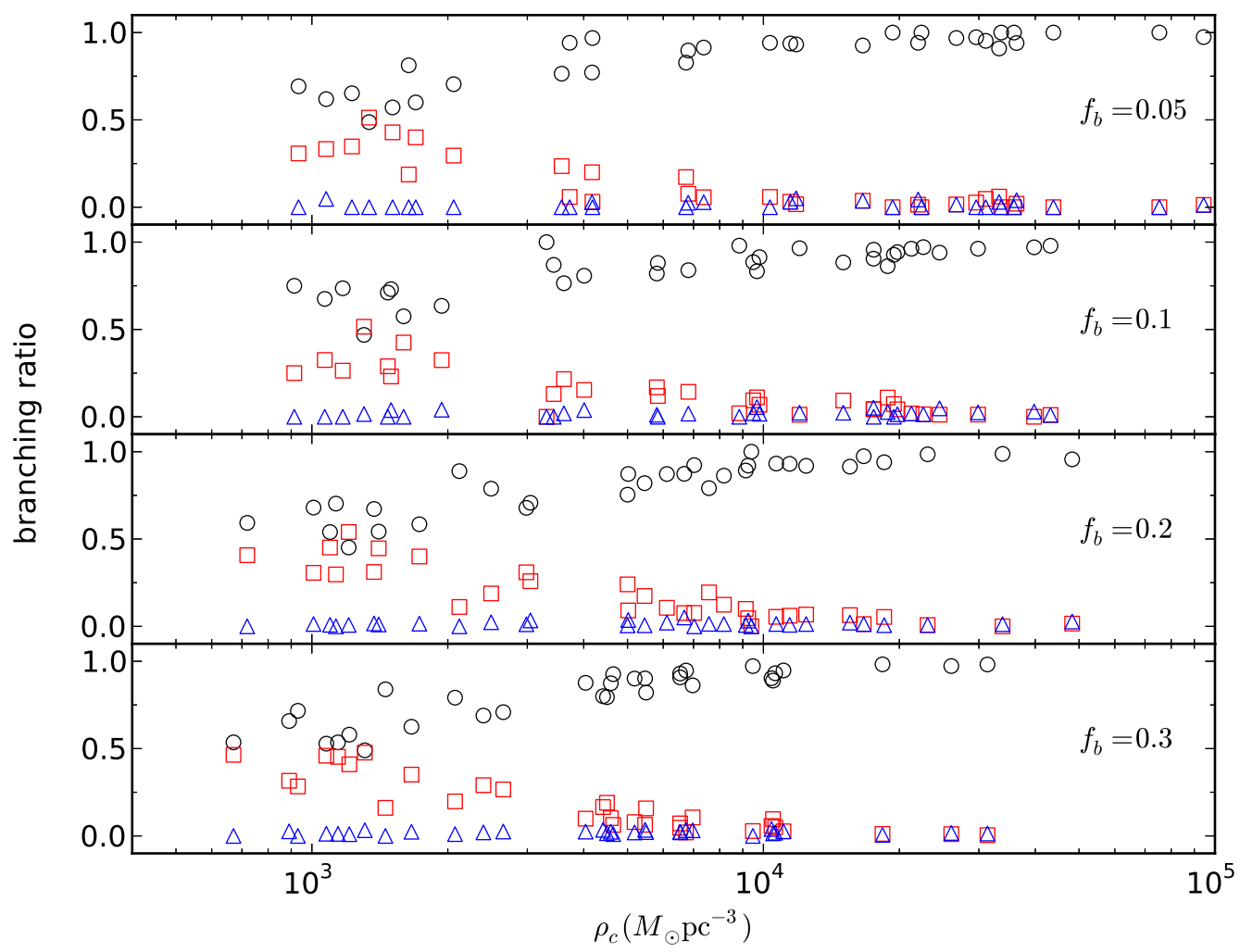

FIG. 5.- Branching ratios of various formation channels for all BSSs in our models vs the respective cluster's $\rho_{c}$. The different panels from top to bottom show subsets of models with different initial $f_{b}$ in an increasing order. The respective initial $f_{b}$ values are shown in each panel. The circles, squares, and triangles denote collisional, MTB, and SE channels, respectively. Clearly, for $\rho_{c}>$ few $\times 10^{3} \mathrm{M}_{\odot} \mathrm{pc}^{-3}$ collisional formations channels dominate for all initial $f_{b}$. BSSs from SE are rare throughout the full range in $\rho_{c}$ and $f_{b}$ in our models. 


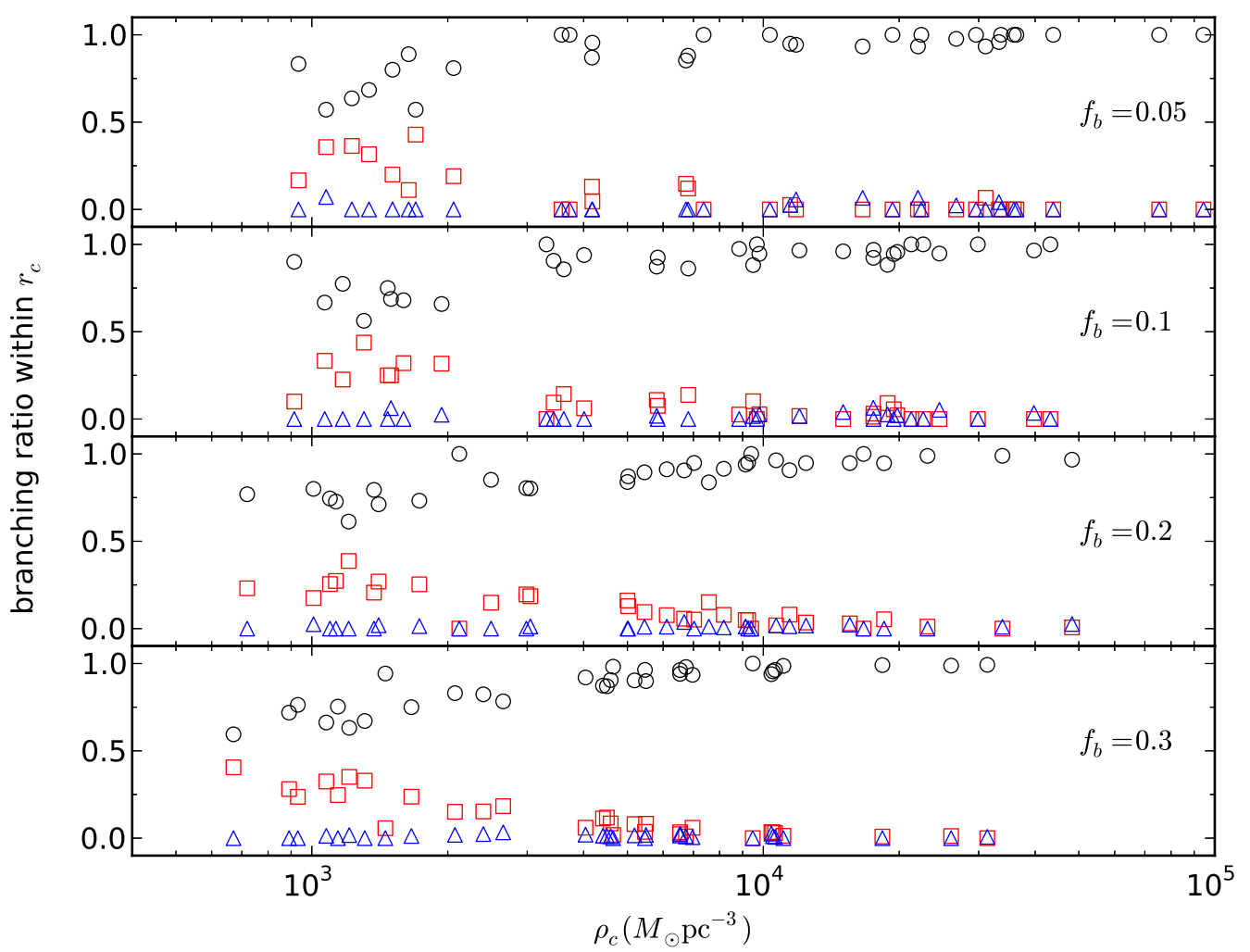

FIG. 6.- Same as Figure 5 but including only the BSSs within the core. Collisional channels dominate the core BSSs throughout the full range in $\rho_{c}$ and $f_{b}$. SE mergers are rare throughout the range of $\rho_{c}$ in our models. MTB channel only becomes comparable to collisional channels if $\rho_{c}<10^{3} \mathrm{M} \odot \mathrm{pc}^{-3}$.

One of the biggest uncertainties in any star-by-star $N$-body model using analytical formulae (Hurlev et al. 2000) for hydrodynamic mergers and stellar evolution is the degree of rejuvenation. Unfortunately, the degree of rejuvenation depends on the detailed kinematics of the encounter (e.g., Sills et al. 1997, 2001) as well as the stellar properties such as rotation, and the ages of the parent stars (Chen \& Han 2009). Incorporating these details is beyond the scope of this study and can only be done via live stellar and binary evolution codes that can rigorously incorporate these physical effects while the whole cluster is dynamically evolving. This is a hard task and has yet to be achieved fully self-consistently by any group. Hence, although the analytical formulae and prescriptions used in BSE (Hurley et al. 2000, 2002) are known to over-estimate the degree of rejuvenation (e.g., Glebbeek \& Pols 2008), for example, for collision products of non-rotating stars, this remains currently the state of the art. For the purpose of this study, however, we expect that the relative contributions from MTB, $\mathrm{SE}$, and various collisional channels should not change by much given that all channels are treated using maximal mixing within BSE, hence rejuvenation may be similarly over-estimated in all cases.

Despite these caveats, it is important to study the distributions of $t_{\text {age }}$ for our model BSSs since they provide us with useful upper limits. The typical distributions of $t_{\text {age }}$ are also interesting in light of the current understanding that most GGCs (those observationally categorized as non-core-collapsed) are still contracting (e.g., Chatterjee et al. 2013). As a result, relevant global cluster properties such as $\rho_{c}, r_{c}$, and $r_{h}$ have not attained steady-state values typical of core-collapsed, "binaryburning" clusters (Chatteriee et al. 2013). Hence, for these contracting clusters it is not sufficient to know the present-day observed cluster properties to fully understand the formation efficiency of BSSs. Instead, the past dynamical history over at least the last $\sim t_{\text {age }}$ should be taken into account. Of course the results (based on the simple full mixing assumption) presented here can also be directly compared with future results from simulations incorporating more sophisticated rejuvenation treatments when they become available.

Figure 10 shows examples of the distributions of $t_{\text {age }}$ for two of our models. These models are chosen such that at least 21 BSSs are created (to ensure enough statistics) and the relative contributions from collisional channels $f_{\text {coll }}$ have extreme values 0.5 (run60), and 1 (run17; Table 1). The central densities differ by a little over an order of magnitude.

The BSSs in the high- $f_{\text {coll }}$ (hence high- $\rho_{c}$ ) model are typically younger compared to those in the low- $f_{\text {coll }}$ model. The broadly peaked distributions seen here are qualitatively similar in all our models. However, the ex- 


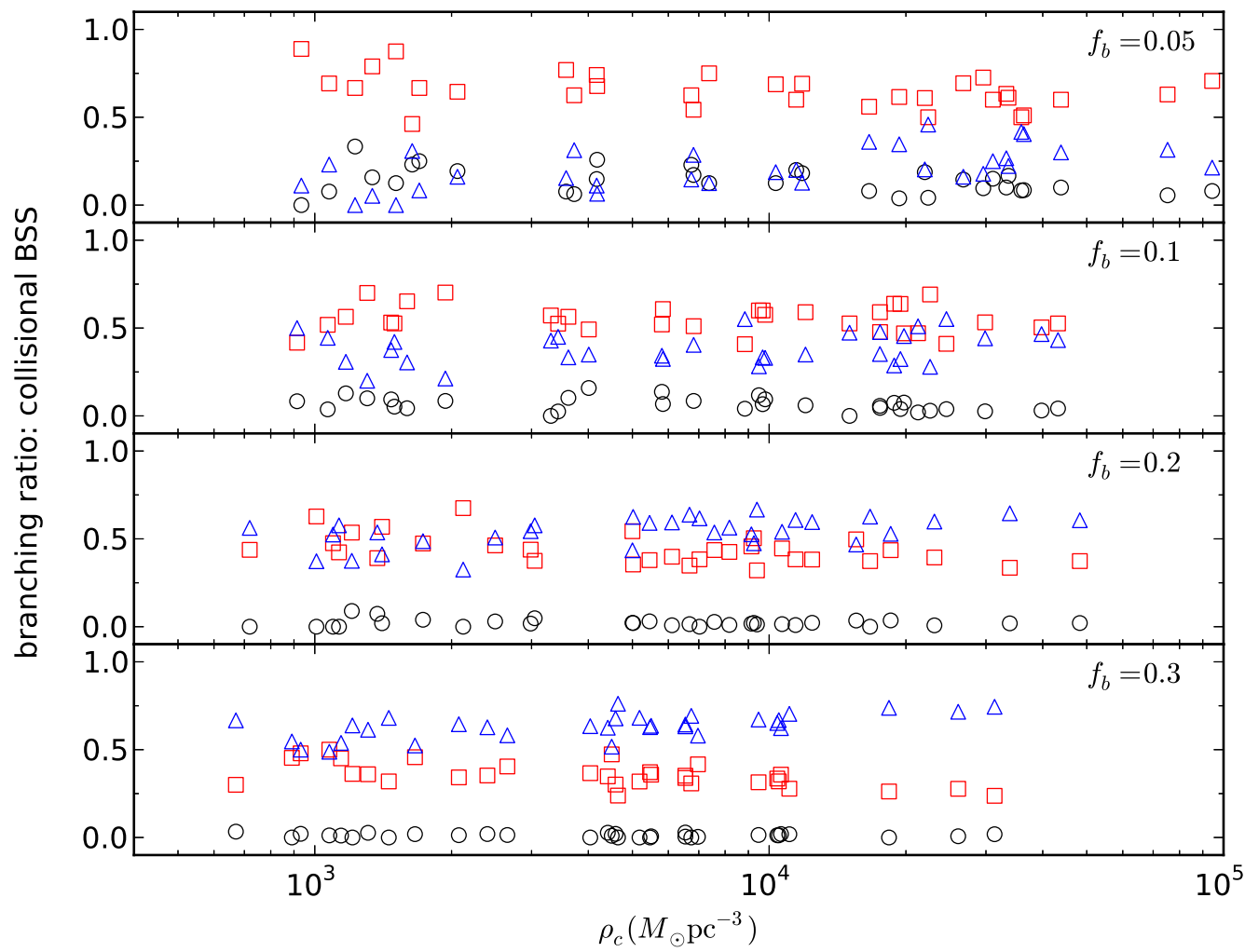

FiG. 7.- Branching ratios between various collisional BSS formation channels vs $\rho_{c}$. Squares, triangles, and circles denote b-s, b-b, and $\mathrm{s}-\mathrm{s}$ collisions, respectively. Each panel shows a subset of models with a given value of initial $f_{b}$ as mentioned on each panel. Throughout the studied range of $\rho_{c}$ and $f_{b}$ contribution from $\mathbf{s}-\mathbf{s}$ channel is minor. Collisions from $\mathrm{b}-\mathrm{s}$ and $\mathrm{b}-\mathrm{b}$ dominate. For high $f_{b}=0.3$ values $\mathrm{b}-\mathrm{b}$ collisions dominate over $\mathrm{b}-\mathrm{s}$ collisions in creation of BSSs.

act positions of these peaks depend on the cluster properties. For the low-density model the bulk of the BSSs have ages between $t_{\text {age }} \approx 0$ and 7 Gyr. In contrast, the BSSs in the high-density model typically have $t_{\text {age }}$ between 0 and 3 Gyr. This is consistent with previous results suggested for the observed BSS population in 47 Tuc (Sills et al. 2000). Half of all BSSs in the low-density model is contained within an age of $\approx 3 \mathrm{Gyr}$, whereas, for the high-density model this value is $\approx 0.9 \mathrm{Gyr}$.

The difference in the peaks of the age distributions between the high and low-density models is expected. In a high-density model the dominant formation channel for BSSs is stellar collisions $(\mathrm{s}-\mathrm{s}, \mathrm{b}-\mathrm{s}$, and $\mathrm{b}-\mathrm{b}$; Figures 4 , 5). Contribution from MTB increases as $\rho_{c}$ decreases (Figure 5). Stellar collisions typically form higher mass BSSs than those formed via MTB. Since a lower mass BSS has a longer residual lifetime than a higher mass counterpart, the typical $t_{\text {age }}$ becomes shorter as $\rho_{c}$ increases due to higher contribution from collisional BSS-formation channels. Indeed we find that the median for the $t_{\text {age }}$ values for the BSSs in our models negatively correlate with $\rho_{c}$ (correlation coefficient $r_{\rho_{c}, t_{\mathrm{age}}}=-0.44$; Figure 11). Due to the same reason the median value of $t_{\text {age }}$ for the core BSSs is typically slightly lower compared to the median value of $t_{\text {age }}$ for all BSSs in a cluster. This effect is more prominent for higher $f_{b}$ models. The number of BSSs outside $r_{c}$ is low for low $f_{b}$ models. Hence, the difference between the median $t_{\text {age }}$ values for BSSs in the whole cluster and in the core decreases with decreasing $f_{b}$. We find that the oldest BSSs in some clusters can be almost as old as the respective clusters.

\section{NUMBER OF CORE BLUE-STRAGGLER STARS VS BINARY FRACTION AND}

After the large-scale surveys using the Hubble Space Telescope (e.g., the ACS survey; Sarajedini et al. 2007; Dotter et al. 2007; Milone et al. 2008) provided high resolution, homogeneously observed and analyzed data for a large number of GGCs, a number of studies have investigated various correlations between the number of BSSs in these clusters and their global properties (e.g., Leigh et al. 2007; Sollima et al. 2008; Knigge et al. 2009; Leigh et al. 2013). In particular, the correlations or lack thereof between $N_{\text {BSS,c }}$ and two cluster properties namely the binary fraction in the core $\left(f_{b, c}\right)$ and the collisional $\Gamma$ generated a lot of interest. Both of these quantities are of great dynamical importance. The presence of primordial binaries increases the BSS formation rates through all channels except $\mathbf{s}-\mathbf{s}$. Instead, only BSS formation via $\mathbf{s}-\mathbf{s}$ is directly related to the collisional $\Gamma$ given by

$$
\Gamma=\left(\frac{\rho_{c}}{M_{\odot} \mathrm{pc}^{-3}}\right)^{2}\left(\frac{r_{c}}{\mathrm{pc}}\right)^{3}\left(\frac{v_{c, \sigma}}{k m s^{-1}}\right)^{-1}
$$




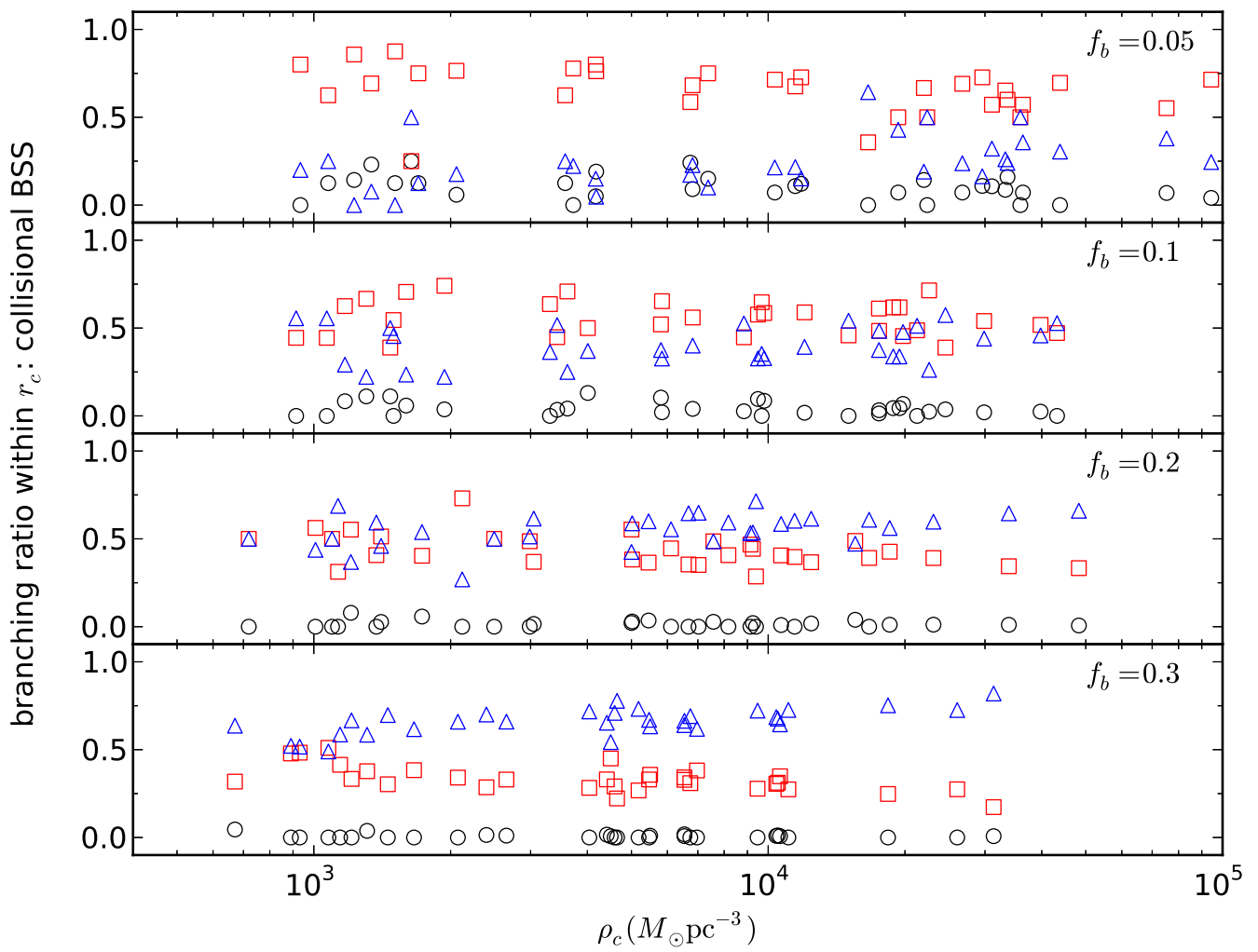

FIG. 8.- Same as Figure 7 but including only the BSSs within the core.

(Pooley \& Hut 2006). After it was found that the numbers of low-mass X-ray binaries and CVs in a cluster are strongly correlated with $\Gamma$, (e.g., Pooley \& Hut 2006) a similar correlation was sought for $N_{\mathrm{BSS}, \mathrm{c}}$ since production of BSSs in the core was also expected to be affected significantly by dynamics. However, it was found that $N_{\text {BSS,c }}$ is rather insensitive to the global $\Gamma$ using data from HST/WFPC2 survey (Piotto et al. 2002). In contrast, it is found that $N_{\mathrm{BSS}, \mathrm{c}}$ shows clear correlation with the number of core binaries in the GGCs (e.g., Leigh et al. 2013). These two pieces of information were inferred as low importance of collisional channels in creation of BSSs in these clusters (e.g., Knigge et al. 2009; Leigh et al. 2013). However, our results suggest that collisions dominate BSS production in the majority of GGCs. We now investigate further this apparent discrepancy.

Figure 12 shows the specific number of BSSs $\left(N_{\mathrm{BSS}} / N_{\mathrm{HB}}\right)$ in our models as a function of the overall $f_{b}$. It also shows $N_{\mathrm{BSS}, \mathrm{c}} / N_{\mathrm{HB}, \mathrm{c}}$ as a function of the core binary fraction $f_{b, c}\left(N_{\mathrm{HB}}\right.$ and $N_{\mathrm{HB}, \mathrm{c}}$ are the numbers of horizontal branch stars in the cluster and in the core, respectively). Clearly, $N_{\mathrm{BSS}} / N_{\mathrm{HB}}$ is correlated with $f_{b}$ and $N_{\mathrm{BSS}, \mathrm{c}} / N_{\mathrm{HB}, \mathrm{c}}$ is correlated with $f_{b, c}$ (spearman correlation coefficient $r_{f_{b, c}, N_{\mathrm{BSS}, \mathrm{c}}}=0.67$ ). For a more detailed comparison with observations see (Sills et al. 2013). This is not at all surprising. Results in Section 3 clearly shows that throughout the studied range in $\rho_{c}$ the dominant BSS production channel is binarymediated collisions ( $\mathrm{b}-\mathrm{s}$ and $\mathrm{b}-\mathrm{b})$. Hence, as $f_{b}$ increases so does the number of BSSs. Although, naturally the number of BSSs from the MTB and SE channels also increases with increasing $f_{b}$, their relative importance is always low compared to $\mathrm{b}-\mathrm{s}$ and $\mathrm{b}-\mathrm{b}$ channels for all $\rho_{c}>10^{3} M_{\odot} \mathrm{pc}^{-3}$. Thus the observed correlation between $N_{\mathrm{BSS}, \mathrm{c}} / N_{\mathrm{HB}, \mathrm{c}}$ with $f_{b, c}$ does not actually indicate BSS formation predominantly via MTB, rather that most collisions are binary-mediated.

Figure 13 shows $N_{\text {BSS,c }}$ as a function of $\Gamma$ calculated using theoretical cluster properties in the code. We find a weak positive correlation between $N_{\mathrm{BSS}, \mathrm{c}}$ and $\Gamma$ (spearman correlation coefficient $r_{\Gamma, N_{\mathrm{BSS}, \mathrm{c}}}=0.12$ ).

The positive correlations of $N_{\mathrm{BSS}, \mathrm{c}}$ with $f_{b, c}$ and $\Gamma$ are qualitatively consistent with the new ACS data (Leigh et al. 2011b, correlation coefficients are 0.4, and 0.5 , respectively). ${ }^{3}$

Other exotic stellar populations, such as low-mass $\mathrm{X}$ ray binaries and cataclysmic variables are thought to be created when compact objects such as neutron stars and white dwarfs collide with giant stars, or acquire

3 Note that the absolute values of the correlation coefficients from models and observed GGCs should not be compared and the comparison here is only qualitative. The unknown priors for the initial conditions used in these models may change these values. In addition, a more stringent comparison requires more observationally motivated determination of BSSs and cluster properties to find $N_{\mathrm{BSS}}, N_{\mathrm{BSS}, \mathrm{c}}, f_{b}, f_{b, c}$, and $\Gamma$. 


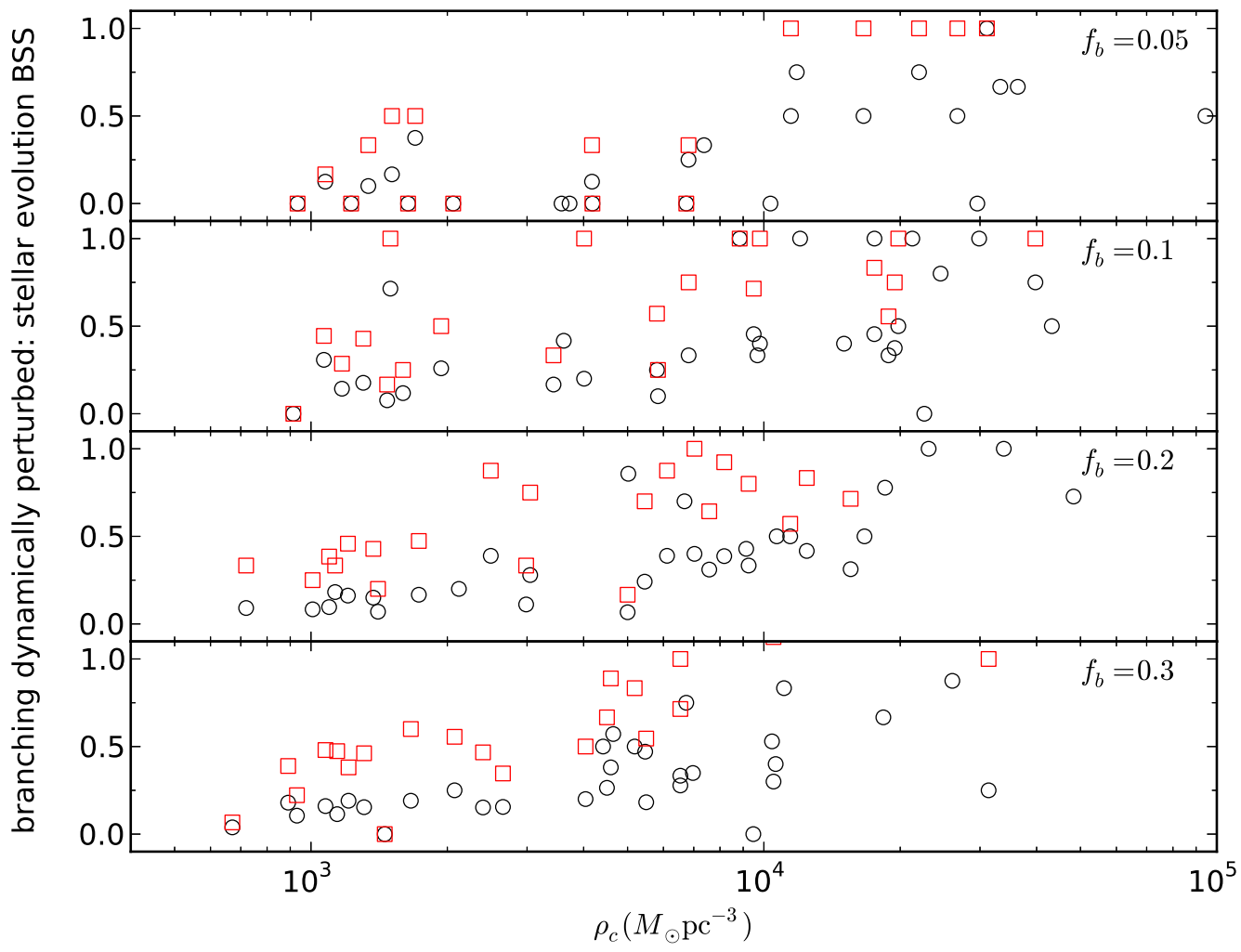

FIG. 9. - Fraction of BSSs created via MTB and SE mergers that had been dynamically perturbed before the change in mass, with respect to all BSSs formed via MTB and SE channels, as a function of $\rho_{c}$. Only perturbations before the production of the BSSs are taken into account. These BSSs would have not been produced with the same properties had there not been a previous encounter. Once produced, these BSSs may interact further; these fractions therefore indicate a lower limit to the fraction of BSSs created via binary stellar evolution and are dynamically altered. Black circles and red squares denote all BSSs in the models and only those within the core, respectively. Each panel from top to bottom show models with initial $\mathrm{fb}=0.05,0.1,0.2$, and 0.3 , respectively.

close binary companions via binary-mediated exchange interactions or tidal captures. Both populations show much tighter correlations with $\Gamma$ compared to $N_{\mathrm{BSS}, \mathrm{c}}$ (Pooley et al. 2003; Pooley \& Hut 2006). We expect this is because binaries with only a narrow range of properties (for example, one star must be a MS star, and the collision product must have mass higher than the $M_{\mathrm{TO}}$ ) create BSSs via collisions. In addition, the collision needs to occur at sufficiently late times such that the generated BSS is still in its residual H-burning stage when the cluster is observed. BSSs produced via MTB, in particular for low-density clusters $\left(\rho_{c}<10^{3} M_{\odot} \mathrm{pc}^{-3}\right)$, also contributes to dilution of the correlation between $N_{\mathrm{BSS} \text {,c }}$ and $\Gamma$. Mass segregation also reduces the number of MS stars in the core compared to relatively massive compact objects at late stages. Hence, production of those populations is more directly related to $\Gamma$ compared to production of BSSs.

\section{CONCLUSIONS}

We have identified the BSSs from a large set of (128) realistic cluster models using a simple and well-defined mass-based criterion. These models use observationally motivated initial conditions, include all relevant physics, and attain properties at $\sim 12$ Gyr including $N, f_{b}, \rho_{c}$, and $r_{c}$ typical of observed GGCs. In addition to cluster dynamical properties, all stellar properties are followed in tandem. For the first time these models allow us to investigate directly the full dynamical and stellar evolution history of each BSS in clusters spanning a large range of parameters. The BSSs identified in these models populate the expected region in a synthetic HRD (Figure 1). In addition, the total numbers, radial distributions (Section 2.3), and trends with various cluster parameters (Section [5] for more detailed comparisons see Sills et al. 2013) are all consistent with the latest observations of BSSs (Leigh et al. 2011b).

We have identified the relative importance of the various relevant formation channels based on the detailed histories of all BSSs formed in our models. We find that collisions play a major role in BSS formation for a large range of central densities typical of GGCs $\left(\rho_{c} \gtrsim\right.$ $10^{3} M_{\odot} \mathrm{pc}^{-3}$; Figures 4-6). BSSs in lower density clusters $\left(\rho_{c} \lesssim 10^{3} M_{\odot} \mathrm{pc}^{-3}\right)$ can have a significant contribution from the MTB channel (up to $\approx 60 \%$ in some of our models). This also indicates that for open cluster densities (typically $<10^{2} \mathrm{M}_{\odot} \mathrm{pc}^{-3}$ ) the dominant channel of BSS formation should be MTB. Binaries are very important for significant production of BSSs in all clusters since most collisions happen via binary-mediated 


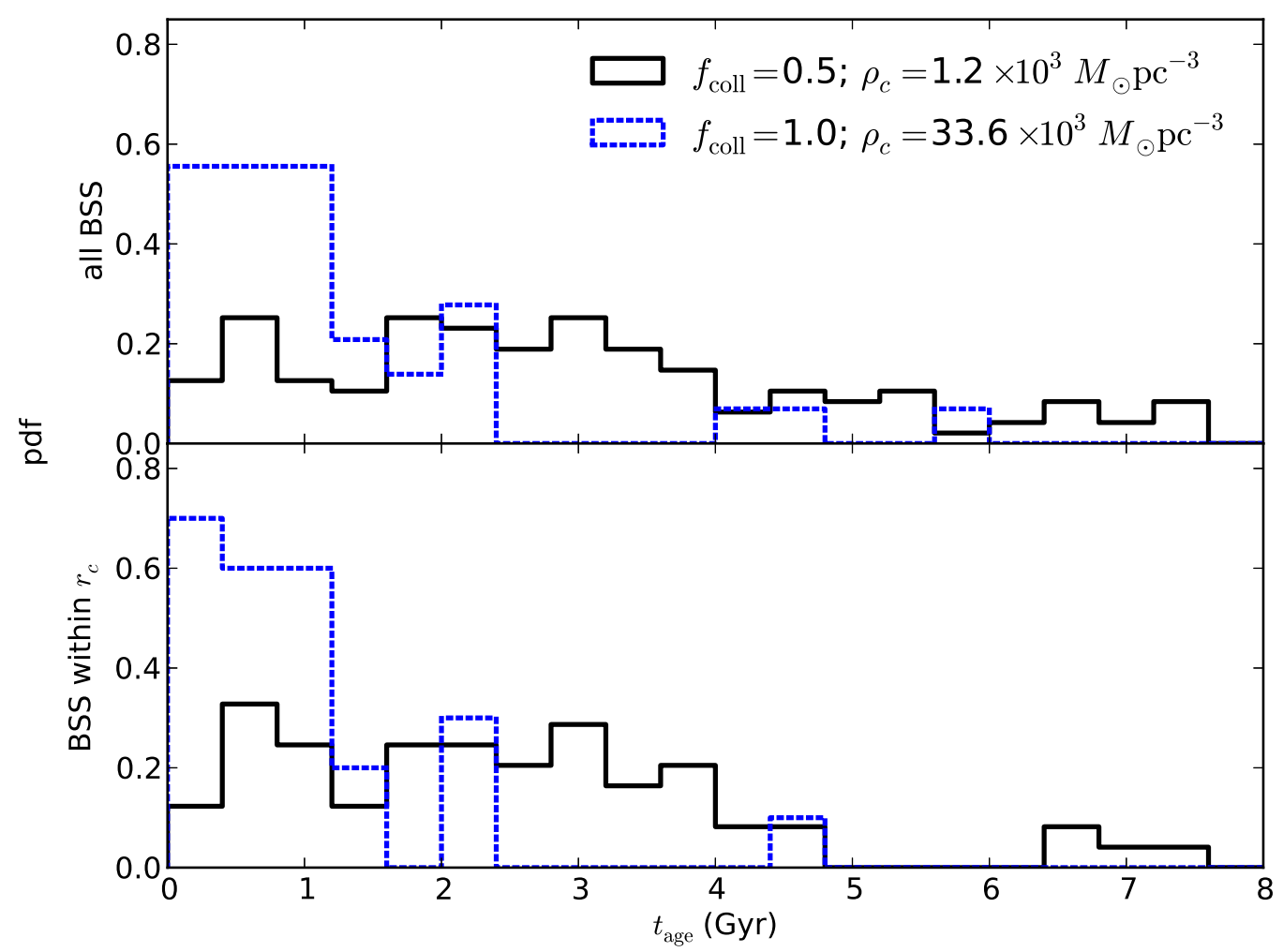

FIG. 10.- Examples for distributions of the time elapsed since formation $\left(t_{\text {age }}\right)$ for the BSSs for two models, one for low (run60) and another for high $\rho_{c}$ (run17; Table 1) with central densities varying by over an order of magnitude. The models were chosen such that each has $N_{\text {BSS }}>21$ and have extreme contributions from collisional channels (0.5 for run60 and 1 for run17). The solid (black) and dashed (blue) lines represent the low and high density clusters, respectively. The top and bottom panels are for all BSSs and those within $r_{c}$, respectively. For MTB BSSs the formation time $t_{\text {form }}$ is set at the beginning of Roche-lobe overflow. The BSSs in the low-density model shows a larger age-spread compared to those in the high-density model. Low-density models have a larger relative contribution from MTB channel. Since MTB BSSs are typically less massive compared to those created via collisions, the residual lifetime for the MTB BSSs is longer than those produced via collisions.

interactions (Figures 77, 8). Even among BSSs created via binary stellar evolution channels (MTB and SE), for $\rho_{c} \gtrsim 10^{4} M_{\odot} \mathrm{pc}^{-3}$ more than $50 \%$ may come from binaries that have been perturbed via dynamical interactions, thereby changing their evolutionary pathways before BSS production. This basic picture could only be changed if, in reality, the degree of mixing from the MTB or SE channels were very different than what is expected from collisions. As an extreme example, if collisions led to no significant mixing, with no fresh Hydrogen supplied to the stellar core, collisions would produce BSSs with much shorter lifetimes. This might increase the relative contribution of MTB in producing the observed BSSs if MTB leads to more mixing and a higher level of rejuvenation. However, as long as collisional channels and MTB produce similar levels of rejuvenation, our results remain at least qualitatively valid.

The importance of binary-mediated collisions in the production of BSSs is of great significance. On the one hand this explains the observed correlation between $N_{\text {BSS,c }}$ and $f_{b, c}$, in particular, in the higher-density $\left(\rho_{c}>\right.$ $10^{3} M_{\odot} \mathrm{pc}^{-3}$ ) GGCs. On the other hand, the importance of collisions suggests that there should be some correla- tion between $N_{\mathrm{BSS}, \mathrm{c}}$ and the global $\Gamma$. Indeed, we find that $N_{\mathrm{BSS}, \mathrm{c}}$ and $\Gamma$ do show some correlation (although weak) for both our models and the GGCs based on the new HST/ACS data with the best available photometry. Early studies (e.g., Davies et al. 2004; Leigh et al. 2007) that claimed no correlation (or anti-correlation) between $N_{\text {BSS,c }}$ and $\Gamma$ used HST/WFPC2 data with lower photometric precision.

Throughout this study we have adopted a simple fullmixing prescription for rejuvenation. Based on this assumption we estimate upper limits for typical $t_{\text {age }}$ for these models. In our models, the oldest BSSs can be almost as old as the host clusters. The typical median ages of the BSSs are, however, between $\approx 1$ and $3 \mathrm{Gyr}$ (depending on $\rho_{c}$; Figure 11 and Table 11). This is consistent with previous estimates for observed BSSs in 47-Tuc (Sills et al. 2000). Thus, present-day BSSs are possibly affected by the dynamical history of their host clusters during the past few billion years. Hence, rate-based studies should take into account the changing global cluster properties during the last $\sim t_{\text {age }}$. Future studies should improve this estimate using more realistic rejuvenation prescriptions via, for example, live single and binary stel- 


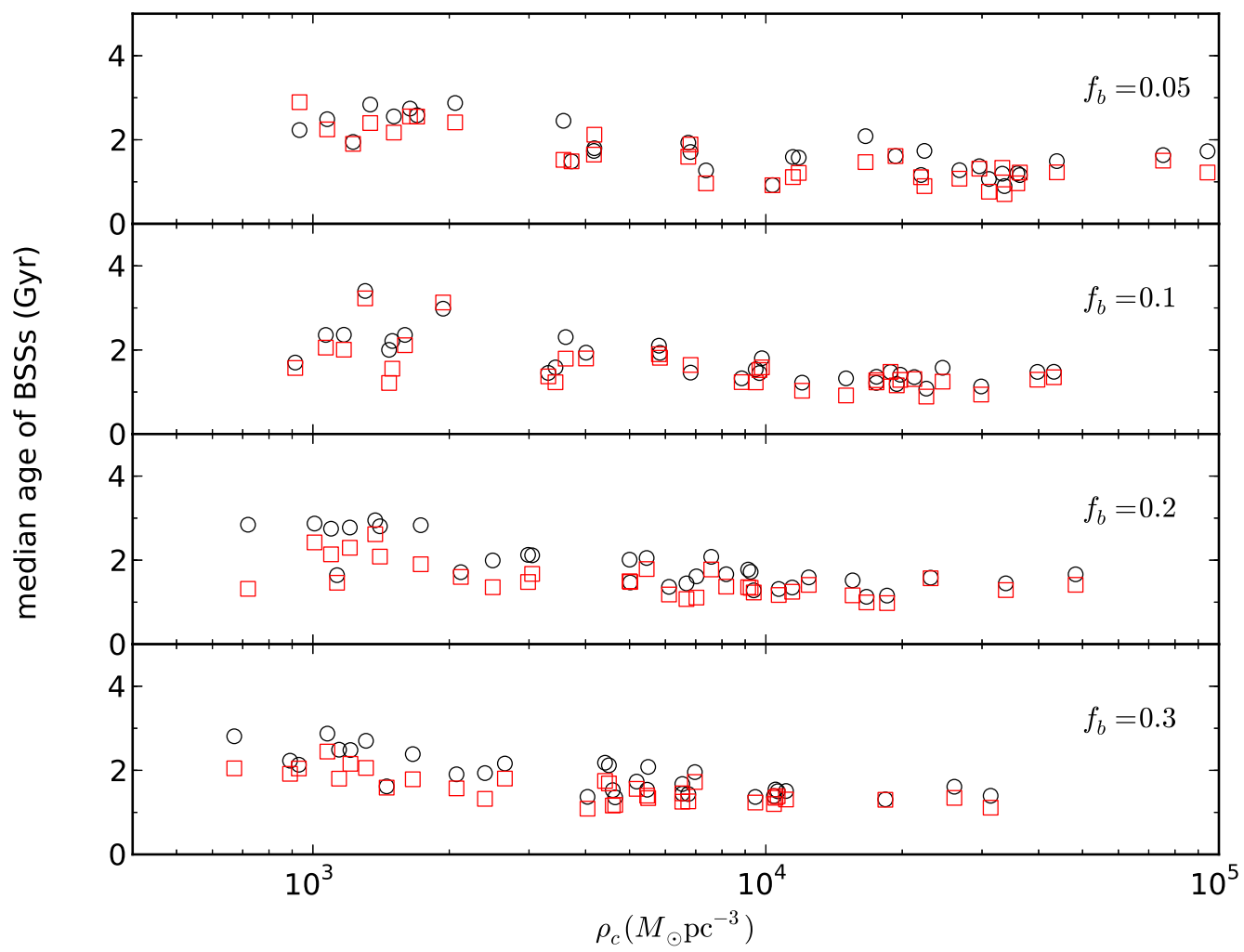

FIG. 11. - The median values for $t_{\text {age }}$ of the BSSs in our models vs $\rho_{c}$. Black circles and red squares denote all BSSs and BSSs in the core, respectively. Top to bottom the panels denote models with initial $f_{b}=0.05,0.1,0.2$, and 0.3 , respectively. The median $t_{\text {age }}$ values for all initial $f_{b}$ show an anti-correlation with $\rho_{c}$. The denser the core, the higher is the contribution from the collisional channels towards BSS production and the lower is the median $t_{\text {age }}$ of the BSSs.

lar evolution codes that can work in parallel with $N$-body codes. However, although, $t_{\text {age }}$ is likely to decrease (e.g., Glebbeek \& Pols 2008), we expect the branching ratios for the various formation channels to remain similar.

\section{ACKNOWLEDGEMENTS}

We thank C. Knigge, N. Leigh, and an anonymous referee for valuable suggestions. Support for this study was provided by NASA through a grant from the Space Telescope Science Institute, which is operated by the Association of Universities for Research in Astronomy, Inc., under NASA contract NAS5-26555; the grant identify- ing number is HST-AR-12829.01-A. SC also acknowledges support from the Department of Astronomy at the University of Florida. FAR acknowledges support from NASA ATP Grant NNX09AO36G at Northwestern University and from NSF Grant PHY-1066293 at the Aspen Center for Physics. AS is supported by the Natural Sciences and Engineering Research Council of Canada. All authors would like to thank the Kavli Institute for Theoretical Physics, where this project began; this work was supported in part at KITP by the National Science Foundation under Grant PHY11-25915.

\section{REFERENCES}

Beccari, G., Lanzoni, B., Ferraro, F. R., Pulone, L., Bellazzini, M., Fusi Pecci, F., Rood, R. T., Giallongo, E., Ragazzoni, R., Grazian, A., Baruffolo, A., Bouche, N., Buschkamp, P., De Santis, C., Diolaiti, E., \& et al. 2008, ApJ, 679, 712

Casertano, S. \& Hut, P. 1985, ApJ, 298, 80

Chatterjee, S., Fregeau, J. M., Umbreit, S., \& Rasio, F. A. 2010, ApJ, 719, 915

Chatterjee, S., Umbreit, S., Fregeau, J. M., \& Rasio, F. A. 2013 MNRAS, 429, 2881

Chen, X. \& Han, Z. 2009, MNRAS, 395, 1822

Dalessandro, E., Lanzoni, B., Ferraro, F. R., Rood, R. T., Milone, A., Piotto, G., \& Valenti, E. 2008a, ApJ, 677, 1069

Dalessandro, E., Lanzoni, B., Ferraro, F. R., Vespe, F., Bellazzini, M., \& Rood, R. T. 2008b, ApJ, 681, 311

Davies, M. B., Piotto, G., \& de Angeli, F. 2004, MNRAS, 349, 129
Davis, D. S., Richer, H. B., Anderson, J., Brewer, J., Hurley, J., Kalirai, J. S., Rich, R. M., \& Stetson, P. B. 2008, AJ, 135, 2155

Djorgovski, S. 1993, in Astronomical Society of the Pacific Conference Series, Vol. 50, Structure and Dynamics of Globular Clusters, ed. S. G. Djorgovski \& G. Meylan, 373

Dotter, A., Chaboyer, B., Jevremović, D., Baron, E., Ferguson, J. W., Sarajedini, A., \& Anderson, J. 2007, AJ, 134, 376

Ferraro, F. R., Fusi Pecci, F., \& Bellazzini, M. 1995, A\&A, 294, 80 Ferraro, F. R., Lanzoni, B., Dalessandro, E., Beccari, G., Pasquato, M., Miocchi, P., Rood, R. T., Sigurdsson, S., Sills, A., Vesperini, E., Mapelli, M., Contreras, R., Sanna, N., \& Mucciarelli, A. 2012, Nature, 492, 393

Fregeau, J. M., Cheung, P., Portegies Zwart, S. F., \& Rasio, F. A. 2004, MNRAS, 352, 1 


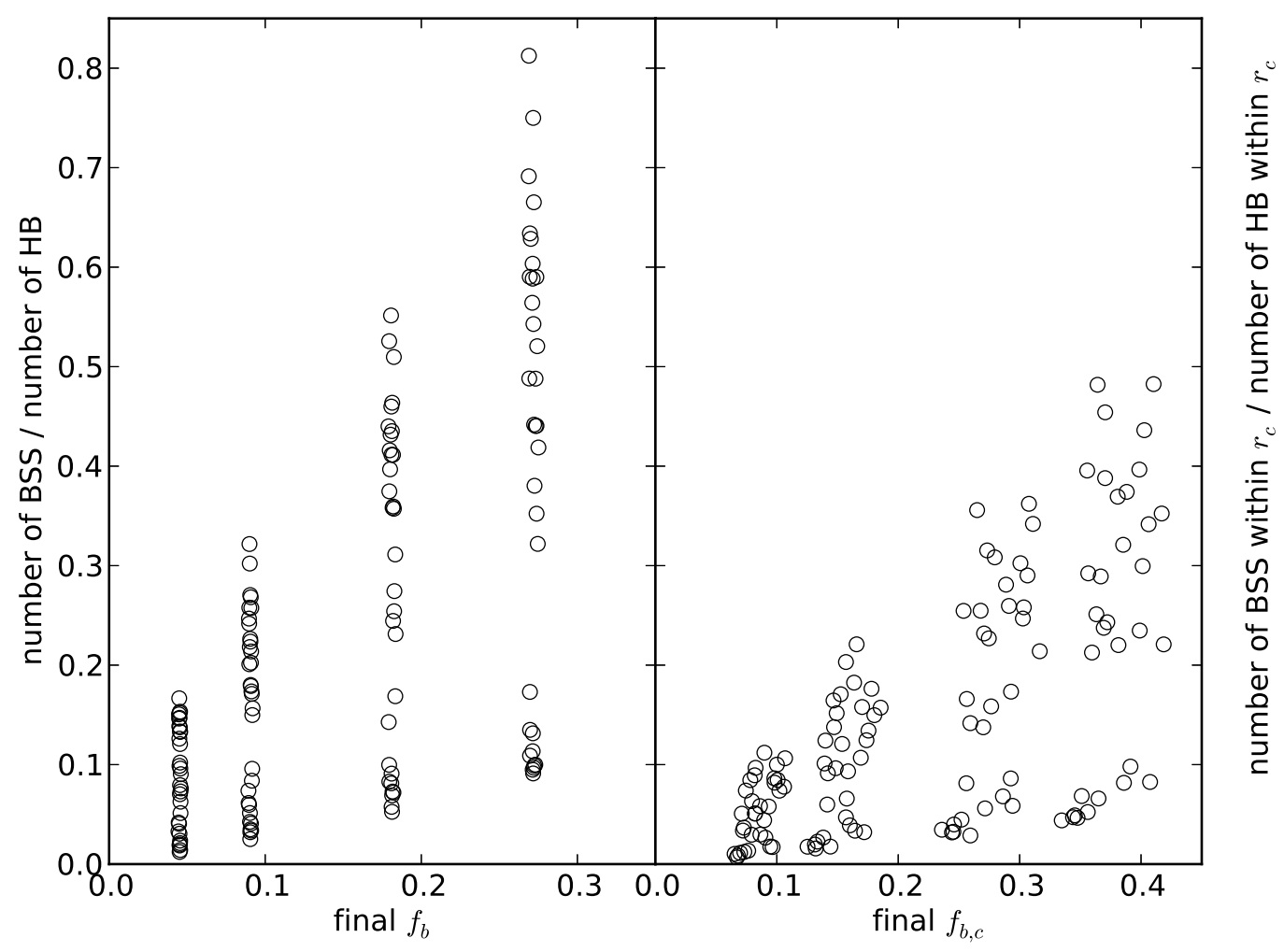

FIG. 12.- Left Panel: The ratio of the number of BSSs $\left(N_{\mathrm{BSS}}\right)$ to the number of horizontal-branch stars $\left(N_{\mathrm{HB}}\right)$ vs the final $f_{b}$. Right Panel: The ratio of the number of core BSSs $\left(N_{\mathrm{BSS}, \mathrm{c}}\right)$ to the number of core horizontal-branch stars $\left(N_{\mathrm{HB}, \mathrm{c}}\right)$ vs the final core binary fraction $\left(f_{b, c}\right)$. The specific BSS frequencies $N_{\mathrm{BSS}} / N_{\mathrm{HB}}$ and $N_{\mathrm{BSS}, \mathrm{c}} / N_{\mathrm{HB}, c}$ are correlated with $f_{b}$ and $f_{b, c}$, respectively. Although the dominant BSS production channel for these models is stellar collisions, most of these collisions are binary mediated. Hence, a higher binary fraction leads to a higher number of collisions and thus a higher number of BSSs if all else is kept unchanged.

Fregeau, J. M., Gürkan, M. A., Joshi, K. J., \& Rasio, F. A. 2003, ApJ, 593, 772

Fregeau, J. M. \& Rasio, F. A. 2007, ApJ, 658, 1047

Fusi Pecci, F., Ferraro, F. R., Corsi, C. E., Cacciari, C., \& Buonanno, R. 1992, AJ, 104, 1831

Giersz, M. \& Heggie, D. C. 2011, MNRAS, 410, 2698

Giersz, M., Heggie, D. C., Hurley, J. R., \& Hypki, A. 2013, MNRAS, 431, 2184

Glebbeek, E. \& Pols, O. R. 2008, A\&A, 488, 1017

Harris, W. E. 1996, AJ, 112, 1487

Heggie, D. \& Hut, P. 2003, The Gravitational Million-Body Problem: A Multidisciplinary Approach to Star Cluster Dynamics (Cambridge University Press, 2003)

Hénon, M. H. 1971, Ap\&SS, 14, 151

Hurley, J. R., Pols, O. R., \& Tout, C. A. 2000, MNRAS, 315, 543

Hurley, J. R., Tout, C. A., \& Pols, O. R. 2002, MNRAS, 329, 897

Hypki, A. \& Giersz, M. 2013, MNRAS, 429, 1221

Joshi, K. J., Nave, C. P., \& Rasio, F. A. 2001, ApJ, 550, 691

Joshi, K. J., Rasio, F. A., \& Portegies Zwart, S. 2000, ApJ, 540, 969

Knigge, C., Leigh, N., \& Sills, A. 2009, Nature, 457, 288

Kozai, Y. 1962, AJ, 67, 591

Kroupa, P. 2001, MNRAS, 322, 231

Leigh, N., Knigge, C., Sills, A., Perets, H. B., Sarajedini, A., \& Glebbeek, E. 2013, MNRAS, 428, 897

Leigh, N., Sills, A., \& Knigge, C. 2007, ApJ, 661, 210

-. 2011a, MNRAS, 416, 1410

—. 2011b, MNRAS, 415, 3771

Lombardi, Jr., J. C., Rasio, F. A., \& Shapiro, S. L. 1995, ApJ, 445, L117

—. 1996, ApJ, 468, 797
Lombardi, Jr., J. C., Warren, J. S., Rasio, F. A., Sills, A., \& Warren, A. R. 2002, ApJ, 568, 939

Mapelli, M., Ripamonti, E., Tolstoy, E., Sigurdsson, S., Irwin, M. J., \& Battaglia, G. 2007, MNRAS, 380, 1127

Mapelli, M., Sigurdsson, S., Ferraro, F. R., Colpi, M., Possenti, A., \& Lanzoni, B. 2006, MNRAS, 373, 361

Mathieu, R. D. \& Geller, A. M. 2009, Nature, 462, 1032

Milone, A. P., Bedin, L. R., Piotto, G., Anderson, J., King, I. R., Sarajedini, A., Dotter, A., Chaboyer, B., Marín-Franch, A., Majewski, S., Aparicio, A., Hempel, M., Paust, N. E. Q., Reid, I. N., Rosenberg, A., \& Siegel, M. 2008, ApJ, 673, 241

Milone, A. P., Piotto, G., Bedin, L. R., Aparicio, A., Anderson, J., Sarajedini, A., Marino, A. F., Moretti, A., Davies, M. B., Chaboyer, B., Dotter, A., Hempel, M., Marín-Franch, A., Majewski, S., Paust, N. E. Q., Reid, I. N., Rosenberg, A., \& Siegel, M. 2012, A\&A, 540, A16

Moretti, A., de Angeli, F., \& Piotto, G. 2008a, VizieR Online Data Catalog, 348, 30183

-. 2008b, A\&A, 483, 183

Pattabiraman, B., Umbreit, S., Liao, W.-k., Choudhary, A.,

Kalogera, V., Memik, G., \& Rasio, F. A. 2013, ApJS, 204, 15

Perets, H. B. \& Fabrycky, D. C. 2009, ApJ, 697, 1048

Piotto, G., King, I. R., Djorgovski, S. G., Sosin, C., Zoccali, M., Saviane, I., de Angeli, F., Riello, M., Recio-Blanco, A., Rich, R. M., Meylan, G., \& Renzini, A. 2002, VizieR Online Data Catalog, 339, 10945

Pooley, D. \& Hut, P. 2006, ApJ, 646, L143

Pooley, D., Lewin, W. H. G., Anderson, S. F., Baumgardt, H., Filippenko, A. V., Gaensler, B. M., Homer, L., Hut, P., Kaspi, V. M., Makino, J., Margon, B., McMillan, S., Portegies Zwart, S., van der Klis, M., \& Verbunt, F. 2003, ApJ, 591, L131 


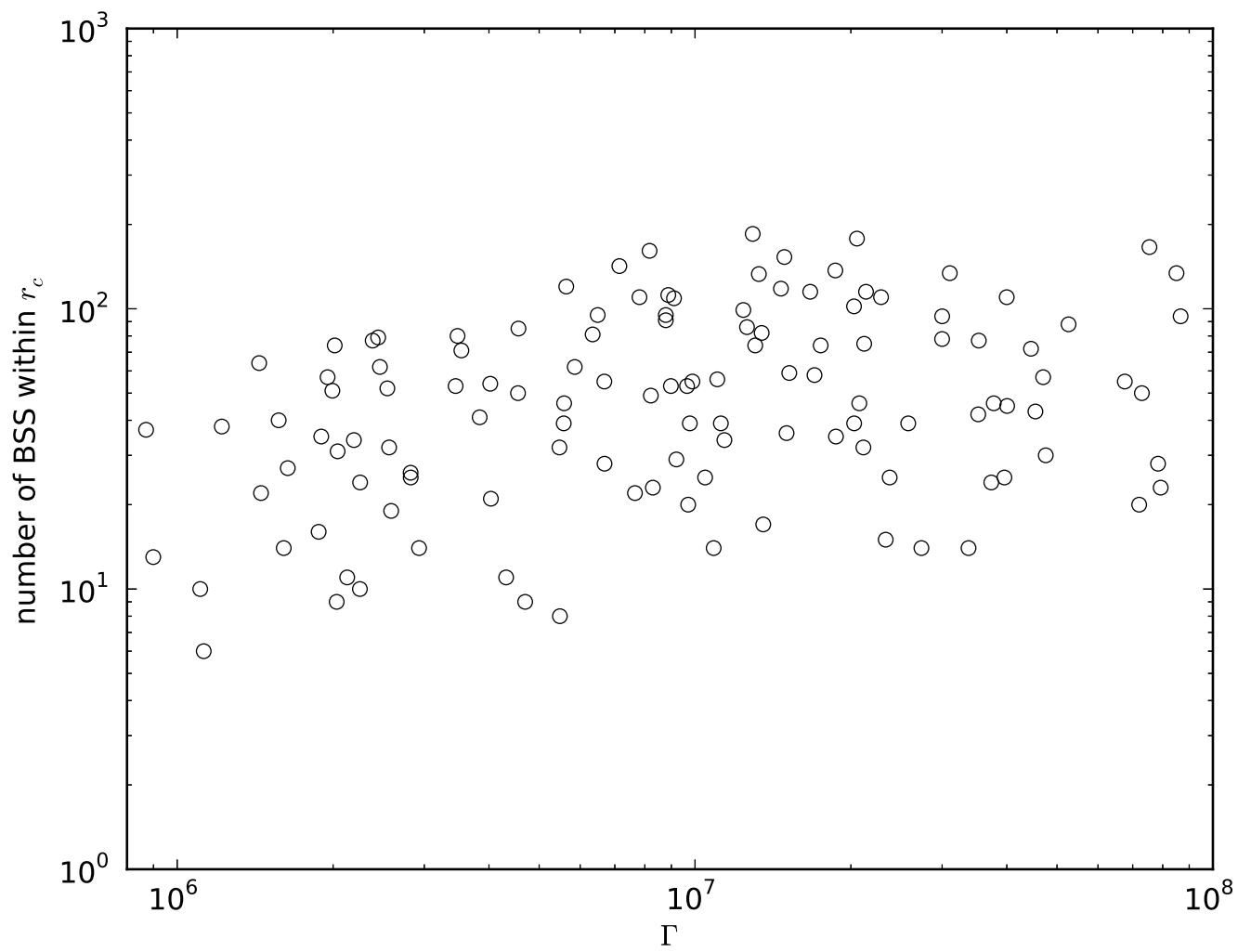

FIg. 13. - $N_{\text {BSS,c }}$ vs $\Gamma$ (Poolev \& Hut 2006) calculated using theoretical definitions in our models using Equation 2 A weak positive correlation (correlation coefficient 0.12 ) between $N_{\mathrm{BSS}, \mathrm{c}}$ and $\Gamma$ is found.

Preston, G. W. \& Sneden, C. 2000, AJ, 120, 1014

Sandage, A. R. 1953, AJ, 58, 61

Sarajedini, A., Bedin, L. R., Chaboyer, B., Dotter, A., Siegel, M., Anderson, J., Aparicio, A., King, I., Majewski, S., Marín-Franch, A., Piotto, G., Reid, I. N., \& Rosenberg, A. 2007, AJ, 133, 1658

Scheepmaker, R. A., Gieles, M., Haas, M. R., Bastian, N., \& Larsen, S. S. The Radii of Thousands of Star Clusters in M51 with HST/ACS, ed. T. Richtler \& S. Larsen, 103

Scheepmaker, R. A., Haas, M. R., Gieles, M., Bastian, N., Larsen, S. S., \& Lamers, H. J. G. L. M. 2007, A\&A, 469, 925

Sills, A., Bailyn, C. D., Edmonds, P. D., \& Gilliland, R. L. 2000, ApJ, 535, 298

Sills, A., Faber, J. A., Lombardi, Jr., J. C., Rasio, F. A., \& Warren, A. R. 2001, ApJ, 548, 323
Sills, A., Glebbeek, E., Chatterjee, S., \& Rasio, F. A. 2013, arXiv:1303.2667 astro-ph.SR

Sills, A., Lombardi, Jr., J. C., Bailyn, C. D., Demarque, P., Rasio, F. A., \& Shapiro, S. L. 1997, ApJ, 487, 290

Sollima, A., Lanzoni, B., Beccari, G., Ferraro, F. R., \& Fusi Pecci, F. 2008, A\&A, 481, 701

Spitzer, L. 1987, Dynamical evolution of globular clusters

Umbreit, S., Fregeau, J. M., Chatterjee, S., \& Rasio, F. A. 2012, ApJ, 750, 31 
TABLE

MOdEL PROPERTIES AND DYNAMICAL PROPERTIES OF BSSS.

\begin{tabular}{|c|c|c|c|c|c|c|c|c|c|c|c|c|c|c|c|c|c|c|c|c|}
\hline Name & $t_{\mathrm{cl}}$ & $M$ & $f_{b}$ & $f_{b, c}$ & $\rho_{c}$ & $\rho_{c, \mathrm{obs}}$ & $r_{c}$ & $c, \mathrm{obs}$ & & & $N_{\mathrm{BS}}$ & & & & & $N_{\mathrm{BSS}}$ & & & $t_{\mathrm{a}}$ & \\
\hline & & & & & & & & & & Coll & & MTB & $\mathrm{SE}$ & & Coll & & TTB & $\mathrm{SE}$ & Med & $\operatorname{Max}$ \\
\hline & & & & & & & & & $s-s$ & $\mathrm{~b}-\mathrm{s}$ & $b-b$ & & & $s-s$ & $\mathrm{~b}-\mathrm{s}$ & $b-b$ & & & & \\
\hline runo & 12 & 14 & 0.05 & 0.09 & 1.03 & 0.29 & 0.95 & 1.43 & 2 & 11 & 3 & 1 & 0 & 1 & 10 & 3 & 0 & 0 & 0.9 & 5.2 \\
\hline run2 & 12 & 21 & 0.05 & 0.09 & 0.74 & 0.09 & 1.23 & 2.97 & 4 & 24 & 4 & 2 & 1 & 3 & 15 & 2 & 0 & 0 & 1.3 & 5.9 \\
\hline run3 & 12 & 28 & 0.05 & 0.08 & 0.68 & 37.00 & 1.39 & 0.08 & 6 & 19 & 10 & 3 & 1 & 2 & 15 & 5 & 3 & 0 & 1.7 & 5.4 \\
\hline run4 & 12 & 36 & 0.05 & 0.07 & 0.67 & 0.89 & 1.50 & 1.41 & 11 & 30 & 7 & 10 & 0 & 7 & 17 & 5 & 5 & 0 & 1.9 & 7.3 \\
\hline run5 & 12 & 14 & 0.09 & 0.17 & 0.88 & 0.20 & 1.02 & 1.93 & 2 & 20 & 27 & 1 & 0 & 1 & 17 & 20 & 1 & 0 & 1.3 & 7.3 \\
\hline run6 & 12 & 22 & 0.09 & 0.16 & 0.68 & 0.97 & 1.27 & 1.00 & 4 & 24 & 19 & 8 & 1 & 1 & 14 & 10 & 4 & 0 & 1.5 & 5.4 \\
\hline run7 & 12 & 29 & 0.09 & 0.15 & 0.58 & 1.02 & 1.46 & 1.14 & 5 & 45 & 24 & 10 & 0 & 1 & 32 & 16 & 4 & 0 & 1.9 & 6.8 \\
\hline run8 & 12 & 36 & 0.09 & 0.14 & 0.58 & 0.22 & 1.58 & 2.66 & 10 & 38 & 25 & 15 & 1 & 5 & 25 & 18 & 6 & 1 & 2.1 & 7.3 \\
\hline run9 & 12 & 15 & 0.18 & 0.29 & 0.50 & 0.85 & 1.23 & 0.82 & 1 & 17 & 30 & 5 & 2 & 1 & 13 & 20 & 5 & 0 & 1.5 & 10.0 \\
\hline run10 & 12 & 23 & 0.18 & 0.29 & 0.61 & 35.43 & 1.34 & 0.09 & 1 & 49 & 73 & 15 & 3 & 0 & 37 & 46 & 7 & 1 & 1.4 & 5.5 \\
\hline run11 & 12 & 30 & 0.18 & 0.27 & 0.55 & 0.45 & 1.52 & 1.73 & 4 & 50 & 78 & 28 & 1 & 3 & 31 & 51 & 9 & 1 & 2.1 & 8.6 \\
\hline run12 & 12 & 38 & 0.18 & 0.25 & 0.50 & 0.19 & 1.68 & 2.70 & 3 & 75 & 60 & 44 & 1 & 2 & 52 & 40 & 18 & 0 & 2.0 & 8.1 \\
\hline run13 & 12 & 16 & 0.27 & 0.40 & 0.40 & 0.20 & 1.32 & 1.85 & 0 & 26 & 45 & 8 & 2 & 0 & 13 & 33 & 3 & 1 & 1.4 & 7.3 \\
\hline run14 & 12 & 24 & 0.27 & 0.39 & 0.46 & 0.52 & 1.47 & 1.39 & 3 & 44 & 99 & 17 & 4 & 0 & 25 & 61 & 8 & 1 & 1.5 & 9.1 \\
\hline run15 & 12 & 32 & 0.27 & 0.37 & 0.44 & 0.46 & 1.64 & 1.59 & 5 & 64 & 115 & 38 & 8 & 2 & 41 & 81 & 16 & 2 & 2.2 & 10.0 \\
\hline run16 & 12 & 40 & 0.27 & 0.36 & 0.45 & 0.18 & 1.76 & 2.98 & 2 & 97 & 106 & 49 & 4 & 1 & 63 & 76 & 19 & 2 & 2.1 & 12.0 \\
\hline run17 & 12 & 14 & 0.05 & 0.11 & 3.36 & 54.35 & 0.67 & 0.09 & 6 & 22 & 8 & 0 & 0 & 4 & 15 & 6 & 0 & 0 & 0.9 & 5.6 \\
\hline run18 & 12 & 21 & 0.05 & 0.09 & 1.66 & 50.28 & 0.95 & 0.07 & 2 & 14 & 9 & 1 & 1 & 0 & 5 & 9 & 0 & 1 & 2.1 & 7.6 \\
\hline run19 & 12 & 28 & 0.04 & 0.08 & 1.18 & 0.33 & 1.17 & 2.00 & 10 & 38 & 7 & 1 & 3 & 4 & 24 & 5 & 0 & 2 & 1.6 & 5.9 \\
\hline run20 & 12 & 36 & 0.04 & 0.08 & 1.15 & 0.11 & 1.27 & 3.43 & 12 & 36 & 12 & 2 & 2 & 4 & 25 & 8 & 1 & 1 & 1.6 & 7.2 \\
\hline run21 & 12 & 14 & 0.09 & 0.19 & 2.13 & 0.49 & 0.78 & 1.05 & 1 & 24 & 26 & 1 & 1 & 0 & 19 & 20 & 0 & 0 & 1.4 & 5.5 \\
\hline run22 & 12 & 22 & 0.09 & 0.16 & 1.20 & 0.22 & 1.06 & 2.18 & 5 & 49 & 29 & 1 & 2 & 1 & 33 & 22 & 1 & 1 & 1.2 & 7.0 \\
\hline run23 & 12 & 29 & 0.09 & 0.15 & 0.95 & 0.36 & 1.26 & 1.83 & 10 & 51 & 24 & 9 & 2 & 5 & 30 & 17 & 6 & 1 & 1.5 & 10.9 \\
\hline run24 & 12 & 36 & 0.09 & 0.15 & 0.98 & 0.94 & 1.34 & 1.36 & 10 & 61 & 35 & 8 & 2 & 6 & 41 & 23 & 2 & 2 & 1.8 & 9.2 \\
\hline run25 & 12 & 15 & 0.18 & 0.30 & 0.94 & 0.69 & 1.01 & 0.99 & 1 & 25 & 52 & 0 & 0 & 0 & 16 & 40 & 0 & 0 & 1.3 & 7.4 \\
\hline run26 & 12 & 23 & 0.18 & 0.29 & 0.91 & 2.27 & 1.18 & 0.73 & 2 & 53 & 61 & 13 & 1 & 0 & 36 & 41 & 4 & 1 & 1.8 & 11.5 \\
\hline run27 & 12 & 30 & 0.18 & 0.27 & 0.76 & 120.61 & 1.37 & 0.10 & 3 & 48 & 59 & 27 & 2 & 2 & 35 & 35 & 13 & 1 & 2.1 & 9.5 \\
\hline run28 & 12 & 38 & 0.18 & 0.26 & 0.82 & 0.56 & 1.44 & 1.71 & 2 & 83 & 110 & 28 & 3 & 0 & 57 & 83 & 12 & 1 & 1.7 & 6.4 \\
\hline run29 & 12 & 16 & 0.27 & 0.40 & 0.52 & 1.98 & 1.22 & 0.52 & 0 & 29 & 62 & 8 & 2 & 0 & 15 & 41 & 5 & 1 & 1.7 & 6.8 \\
\hline run30 & 12 & 24 & 0.27 & 0.39 & 0.55 & 0.33 & 1.39 & 1.67 & 0 & 58 & 98 & 11 & 6 & 0 & 35 & 71 & 4 & 0 & 1.5 & 7.5 \\
\hline run31 & 12 & 32 & 0.27 & 0.37 & 0.55 & 0.81 & 1.53 & 1.16 & 1 & 54 & 96 & 29 & 4 & 1 & 35 & 62 & 9 & 2 & 2.1 & 8.0 \\
\hline run32 & 12 & 40 & 0.27 & 0.36 & 0.70 & 7.71 & 1.53 & 0.37 & 1 & 112 & 156 & 33 & 10 & 0 & 66 & 107 & 11 & 1 & 2.0 & 11.1 \\
\hline run33* & 11 & 14 & 0.05 & 0.10 & 3.33 & 379.65 & 0.66 & 0.06 & 3 & 19 & 8 & 2 & 1 & 2 & 15 & 6 & 0 & 1 & 1.2 & 5.2 \\
\hline run34 & 11 & 21 & 0.05 & 0.10 & 3.11 & 4.46 & 0.79 & 0.61 & 6 & 24 & 10 & 2 & 0 & 3 & 16 & 9 & 2 & 0 & 1.1 & 3.9 \\
\hline run35 & 11 & 29 & 0.04 & 0.09 & 2.67 & 3.25 & 0.91 & 0.67 & 9 & 43 & 10 & 1 & 1 & 3 & 29 & 10 & 0 & 1 & 1.3 & 5.8 \\
\hline run36 & 11 & 36 & 0.04 & 0.08 & 2.20 & 1.50 & 1.03 & 1.17 & 12 & 39 & 13 & 1 & 3 & 6 & 28 & 8 & 0 & 3 & 1.2 & 5.2 \\
\hline run37 & 11 & 14 & 0.09 & 0.17 & 1.75 & 59.93 & 0.82 & 0.07 & 2 & 21 & 21 & 2 & 0 & 1 & 15 & 15 & 1 & 0 & 1.4 & 6.5 \\
\hline run38 & 11 & 22 & 0.09 & 0.18 & 2.26 & 0.49 & 0.89 & 1.56 & 2 & 47 & 19 & 1 & 1 & 1 & 30 & 11 & 0 & 0 & 1.1 & 5.7 \\
\hline run39 & 11 & 29 & 0.09 & 0.16 & 1.75 & 2.44 & 1.05 & 0.76 & 6 & 62 & 37 & 5 & 6 & 1 & 44 & 27 & 1 & 5 & 1.2 & 6.8 \\
\hline run40 & 11 & 37 & 0.09 & 0.15 & 1.88 & 91.50 & 1.09 & 0.08 & 7 & 60 & 27 & 12 & 3 & 3 & 42 & 23 & 7 & 2 & 1.5 & 9.1 \\
\hline run41 & 11 & 15 & 0.18 & 0.32 & 1.67 & 0.55 & 0.85 & 1.44 & 0 & 28 & 47 & 1 & 1 & 0 & 18 & 28 & 0 & 0 & 1.1 & 5.6 \\
\hline run42 & 11 & 23 & 0.18 & 0.31 & 1.85 & 0.83 & 0.96 & 1.07 & 5 & 61 & 74 & 8 & 1 & 1 & 38 & 50 & 5 & 0 & 1.2 & 8.8 \\
\hline run43 & 11 & 30 & 0.18 & 0.28 & 1.24 & 1.15 & 1.17 & 1.08 & 3 & 52 & 81 & 10 & 2 & 2 & 40 & 67 & 4 & 2 & 1.6 & 6.0 \\
\hline run44 & 12 & 38 & 0.18 & 0.27 & 1.55 & 1.23 & 1.19 & 1.23 & 6 & 85 & 80 & 12 & 4 & 5 & 62 & 60 & 4 & 3 & 1.5 & 7.1 \\
\hline run45 & 12 & 15 & 0.27 & 0.42 & 1.11 & 1.15 & 0.95 & 0.73 & 2 & 30 & 76 & 3 & 3 & 0 & 20 & 53 & 1 & 0 & 1.5 & 7.2 \\
\hline run46 & 11 & 24 & 0.27 & 0.40 & 1.04 & 4.31 & 1.15 & 0.45 & 2 & 53 & 103 & 10 & 7 & 1 & 33 & 74 & 4 & 3 & 1.4 & 6.8 \\
\hline run47 & 12 & 32 & 0.27 & 0.38 & 1.06 & 0.97 & 1.25 & 1.09 & 4 & 73 & 127 & 11 & 4 & 1 & 46 & 85 & 4 & 1 & 1.5 & 10.0 \\
\hline run48 & 12 & 40 & 0.27 & 0.37 & 1.05 & 0.51 & 1.35 & 1.69 & 3 & 77 & 161 & 26 & 4 & 2 & 53 & 115 & 6 & 2 & 1.5 & 9.6 \\
\hline run49 & 12 & 14 & 0.05 & 0.07 & 0.09 & 0.19 & 2.11 & 1.72 & 0 & 8 & 1 & 4 & 0 & 0 & 4 & 1 & 1 & 0 & 2.2 & 5.2 \\
\hline run50 & 12 & 21 & 0.05 & 0.07 & 0.11 & 0.03 & 2.32 & 4.19 & 1 & 9 & 3 & 7 & 1 & 1 & 5 & 2 & 5 & 1 & 2.5 & 7.5 \\
\hline run51 & 12 & 29 & 0.05 & 0.07 & 0.12 & 0.06 & 2.45 & 3.95 & 5 & 10 & 0 & 8 & 0 & 1 & 6 & 0 & 4 & 0 & 1.9 & 7.4 \\
\hline run52 & 12 & 36 & 0.05 & 0.07 & 0.13 & 0.20 & 2.56 & 2.49 & 3 & 15 & 1 & 20 & 0 & 3 & 9 & 1 & 6 & 0 & 2.8 & 7.3 \\
\hline run53 & 12 & 14 & 0.09 & 0.14 & 0.09 & 0.04 & 2.14 & 3.29 & 1 & 5 & 6 & 4 & 0 & 0 & 4 & 5 & 1 & 0 & 1.7 & 4.9 \\
\hline run54 & 12 & 22 & 0.09 & 0.14 & 0.11 & 0.10 & 2.35 & 2.54 & 1 & 14 & 12 & 13 & 0 & 0 & 8 & 10 & 9 & 0 & 2.4 & 6.6 \\
\hline run55 & 12 & 29 & 0.09 & 0.13 & 0.12 & 0.03 & 2.49 & 4.70 & 5 & 22 & 12 & 14 & 0 & 2 & 15 & 7 & 7 & 0 & 2.4 & 7.5 \\
\hline run56 & 12 & 37 & 0.09 & 0.13 & 0.13 & 2.82 & 2.60 & 0.31 & 3 & 21 & 6 & 33 & 1 & 2 & 12 & 4 & 14 & 0 & 3.4 & 8.7 \\
\hline run57 & 12 & 15 & 0.18 & 0.26 & 0.07 & 2.43 & 2.34 & 0.17 & 0 & 7 & 9 & 11 & 0 & 0 & 5 & 5 & 3 & 0 & 2.8 & 6.1 \\
\hline run58 & 12 & 23 & 0.18 & 0.26 & 0.10 & 0.16 & 2.41 & 2.13 & 0 & 32 & 19 & 23 & 1 & 0 & 18 & 14 & 7 & 1 & 2.9 & 7.4 \\
\hline run59 & 12 & 31 & 0.18 & 0.25 & 0.11 & 0.20 & 2.59 & 2.02 & 0 & 29 & 32 & 51 & 1 & 0 & 19 & 19 & 13 & 0 & 2.7 & 10.4 \\
\hline run60 & 12 & 38 & 0.18 & 0.24 & 0.12 & 0.04 & 2.70 & 5.07 & 5 & 30 & 21 & 67 & 1 & 3 & 21 & 14 & 24 & 0 & 2.8 & 10.4 \\
\hline run61 & 12 & 16 & 0.27 & 0.36 & 0.07 & 5.88 & 2.43 & 0.17 & 1 & 9 & 20 & 26 & 0 & 1 & 7 & 14 & 15 & 0 & 2.8 & 5.6 \\
\hline run62 & 12 & 24 & 0.27 & 0.36 & 0.09 & 3.78 & 2.55 & 0.17 & 0 & 34 & 41 & 36 & 3 & 0 & 22 & 24 & 18 & 0 & 2.2 & 7.3 \\
\hline run63 & 12 & 32 & 0.27 & 0.35 & 0.11 & 0.12 & 2.64 & 2.99 & 1 & 42 & 41 & 73 & 2 & 0 & 25 & 24 & 24 & 1 & 2.9 & 11.8 \\
\hline run64 & 12 & 40 & 0.27 & 0.33 & 0.11 & 0.06 & 2.78 & 4.07 & 1 & 41 & 49 & 77 & 2 & 0 & 24 & 34 & 19 & 0 & 2.5 & 10.6 \\
\hline run65 & 12 & 14 & 0.05 & 0.08 & 0.16 & 0.09 & 1.77 & 2.17 & 3 & 6 & 4 & 3 & 0 & 2 & 2 & 4 & 1 & 0 & 2.7 & 5.2 \\
\hline run66 & 12 & 21 & 0.05 & 0.07 & 0.15 & 2.84 & 2.07 & 0.25 & 1 & 7 & 0 & 6 & 0 & 1 & 7 & 0 & 2 & 0 & 2.6 & 6.3 \\
\hline run67 & 12 & 29 & 0.05 & 0.07 & 0.17 & 0.07 & 2.19 & 3.45 & 3 & 8 & 1 & 8 & 0 & 1 & 6 & 1 & 6 & 0 & 2.6 & 8.5 \\
\hline run68 & 12 & 36 & 0.05 & 0.07 & 0.21 & 0.06 & 2.22 & 4.26 & 6 & 20 & 5 & 13 & 0 & 1 & 13 & 3 & 4 & 0 & 2.9 & 7.0 \\
\hline run69 & 12 & 14 & 0.09 & 0.15 & 0.15 & 0.13 & 1.83 & 2.21 & 1 & 10 & 8 & 6 & 1 & 0 & 6 & 5 & 4 & 1 & 2.2 & 6.6 \\
\hline run70 & 12 & 22 & 0.09 & 0.14 & 0.15 & 5.07 & 2.10 & 0.14 & 3 & 17 & 12 & 13 & 0 & 2 & 7 & 9 & 6 & 0 & 2.0 & 6.9 \\
\hline run71 & 12 & 29 & 0.09 & 0.13 & 0.16 & 5.08 & 2.25 & 0.12 & 1 & 15 & 7 & 17 & 0 & 1 & 12 & 4 & 8 & 0 & 2.4 & 10.5 \\
\hline run72 & 12 & 37 & 0.09 & 0.13 & 0.19 & 0.08 & 2.28 & 3.55 & 4 & 33 & 10 & 24 & 3 & 1 & 20 & 6 & 13 & 1 & 3.0 & 11.3 \\
\hline run73 & 12 & 15 & 0.18 & 0.27 & 0.11 & 0.11 & 2.03 & 2.06 & 0 & 11 & 15 & 11 & 0 & 0 & 5 & 11 & 6 & 0 & 1.6 & 8.2 \\
\hline run74 & 12 & 23 & 0.18 & 0.26 & 0.14 & 0.06 & 2.19 & 3.42 & 3 & 16 & 22 & 19 & 1 & 0 & 11 & 16 & 7 & 0 & 2.9 & 7.2 \\
\hline
\end{tabular}


TABLE 1 - Continued

\begin{tabular}{|c|c|c|c|c|c|c|c|c|c|c|c|c|c|c|c|c|c|c|c|c|}
\hline \multirow[t]{3}{*}{ Name } & \multirow[t]{3}{*}{$t_{\mathrm{cl}}$} & \multirow[t]{3}{*}{$M$} & \multirow[t]{3}{*}{$\overline{f_{b}}$} & \multirow{3}{*}{$f_{b, c}$} & \multirow[t]{3}{*}{$\rho_{c}$} & \multirow{3}{*}{$\rho_{c, \mathrm{obs}}$} & \multirow[t]{3}{*}{$r_{c}$} & \multirow[t]{3}{*}{$r_{c, \mathrm{obs}}$} & \multicolumn{5}{|c|}{$N_{\mathrm{BSS}}$} & & & $N_{\mathrm{BSS}}$ & & & $t_{c}$ & \\
\hline & & & & & & & & & & Coll & & MTB & $\mathrm{SE}$ & & Coll & & МТВ & $\mathrm{SE}$ & Med & $\operatorname{Max}$ \\
\hline & & & & & & & & & $s-s$ & $b-s$ & $\mathrm{~b}-\mathrm{b}$ & & & $s-s$ & $\mathrm{~b}-\mathrm{s}$ & $\mathrm{b}-\mathrm{b}$ & & & & \\
\hline run75 & 12 & 30 & 0.18 & 0.24 & 0.14 & 0.07 & 2.38 & 3.49 & 1 & 29 & 21 & 42 & 1 & 1 & 19 & 17 & 14 & 1 & 2.8 & 6.9 \\
\hline run76 & 12 & 38 & 0.18 & 0.25 & 0.17 & 0.14 & 2.40 & 2.97 & 3 & 36 & 37 & 52 & 2 & 3 & 21 & 28 & 18 & 1 & 2.8 & 10.0 \\
\hline run77 & 12 & 16 & 0.28 & 0.37 & 0.09 & 0.05 & 2.18 & 3.01 & 1 & 23 & 24 & 19 & 0 & 0 & 14 & 15 & 9 & 0 & 2.1 & 8.2 \\
\hline run78 & 12 & 24 & 0.27 & 0.36 & 0.12 & 7.73 & 2.30 & 0.13 & 0 & 21 & 37 & 41 & 1 & 0 & 12 & 24 & 20 & 1 & 2.5 & 11.7 \\
\hline run79 & 12 & 32 & 0.27 & 0.35 & 0.13 & 0.13 & 2.46 & 2.68 & 2 & 27 & 46 & 73 & 5 & 2 & 20 & 31 & 26 & 0 & 2.7 & 9.5 \\
\hline run80 & 12 & 40 & 0.27 & 0.34 & 0.17 & 0.13 & 2.46 & 2.98 & 2 & 48 & 55 & 59 & 4 & 0 & 23 & 37 & 19 & 1 & 2.4 & 7.4 \\
\hline run81 & 12 & 14 & 0.05 & 0.08 & 0.37 & 0.23 & 1.35 & 1.54 & 1 & 10 & 5 & 1 & 0 & 0 & 7 & 2 & 0 & 0 & 1.5 & 5.2 \\
\hline run82 & 12 & 21 & 0.05 & 0.08 & 0.36 & 0.32 & 1.57 & 1.45 & 1 & 10 & 2 & 4 & 0 & 1 & 5 & 2 & 0 & 0 & 2.5 & 7.0 \\
\hline run83 & 12 & 28 & 0.05 & 0.08 & 0.42 & 0.23 & 1.65 & 2.18 & 8 & 21 & 2 & 1 & 0 & 4 & 16 & 1 & 1 & 0 & 1.8 & 5.2 \\
\hline run84 & 12 & 36 & 0.05 & 0.07 & 0.42 & 0.13 & 1.76 & 3.01 & 4 & 20 & 3 & 7 & 1 & 1 & 16 & 3 & 3 & 0 & 1.7 & 6.3 \\
\hline run85 & 12 & 14 & 0.09 & 0.16 & 0.33 & 0.61 & 1.42 & 0.81 & 0 & 8 & 6 & 0 & 0 & 0 & 7 & 4 & 0 & 0 & 1.5 & 6.1 \\
\hline run86 & 12 & 22 & 0.09 & 0.15 & 0.34 & 0.20 & 1.61 & 1.99 & 1 & 21 & 18 & 6 & 0 & 1 & 13 & 15 & 3 & 0 & 1.6 & 10.5 \\
\hline run87 & 12 & 29 & 0.09 & 0.14 & 0.36 & 0.14 & 1.74 & 2.76 & 4 & 22 & 13 & 11 & 1 & 1 & 17 & 6 & 4 & 0 & 2.3 & 10.5 \\
\hline run88 & 12 & 36 & 0.09 & 0.14 & 0.40 & 0.24 & 1.81 & 2.25 & 10 & 31 & 22 & 12 & 3 & 6 & 23 & 17 & 3 & 0 & 1.9 & 7.0 \\
\hline run89 & 12 & 15 & 0.18 & 0.28 & 0.21 & 0.16 & 1.66 & 1.96 & 0 & 27 & 13 & 5 & 0 & 0 & 19 & 7 & 0 & 0 & 1.7 & 5.2 \\
\hline run90 & 12 & 23 & 0.18 & 0.27 & 0.25 & 0.19 & 1.80 & 2.00 & 2 & 31 & 34 & 16 & 2 & 0 & 23 & 23 & 8 & 0 & 2.0 & 7.5 \\
\hline run91 & 12 & 30 & 0.18 & 0.26 & 0.30 & 0.12 & 1.86 & 2.78 & 1 & 25 & 31 & 26 & 1 & 0 & 18 & 19 & 9 & 0 & 2.1 & 6.7 \\
\hline run92 & 12 & 38 & 0.18 & 0.25 & 0.30 & 0.33 & 1.99 & 1.90 & 5 & 39 & 60 & 38 & 5 & 1 & 24 & 40 & 15 & 1 & 2.1 & 7.8 \\
\hline run93 & 12 & 16 & 0.27 & 0.38 & 0.15 & 0.22 & 1.87 & 1.36 & 0 & 15 & 32 & 9 & 0 & 0 & 10 & 23 & 2 & 0 & 1.6 & 6.9 \\
\hline run94 & 12 & 24 & 0.27 & 0.37 & 0.21 & 1.11 & 1.93 & 0.76 & 1 & 26 & 49 & 19 & 1 & 0 & 15 & 29 & 8 & 1 & 1.9 & 7.2 \\
\hline run95 & 12 & 32 & 0.27 & 0.36 & 0.24 & 0.17 & 2.02 & 2.26 & 2 & 36 & 64 & 43 & 3 & 1 & 20 & 49 & 13 & 2 & 1.9 & 8.1 \\
\hline run96 & 12 & 40 & 0.27 & 0.35 & 0.27 & 27.80 & 2.11 & 0.11 & 2 & 57 & 82 & 53 & 5 & 1 & 31 & 62 & 22 & 4 & 2.2 & 7.5 \\
\hline run97* & 12 & 14 & 0.05 & 0.11 & 2.24 & 0.27 & 0.79 & 1.36 & 1 & 12 & 11 & 0 & 0 & 0 & 7 & 7 & 0 & 0 & 1.7 & 6.8 \\
\hline run98 & 12 & 21 & 0.05 & 0.10 & 3.59 & 11.10 & 0.79 & 0.24 & 3 & 18 & 15 & 0 & 0 & 0 & 10 & 10 & 0 & 0 & 1.2 & 7.5 \\
\hline run99 & 12 & 28 & 0.05 & 0.10 & 3.64 & 65.46 & 0.85 & 0.07 & 4 & 24 & 19 & 1 & 2 & 2 & 16 & 10 & 0 & 0 & 1.2 & 4.3 \\
\hline run100 & 12 & 36 & 0.04 & 0.09 & 2.96 & 1.33 & 0.96 & 0.95 & 7 & 53 & 13 & 2 & 0 & 6 & 40 & 9 & 0 & 0 & 1.4 & 6.2 \\
\hline run101* & 12 & 14 & 0.09 & 0.18 & 1.50 & 21.45 & 0.91 & 0.09 & 0 & 20 & 18 & 4 & 1 & 0 & 11 & 13 & 0 & 1 & 1.3 & 5.2 \\
\hline run102* & 12 & 22 & 0.09 & 0.18 & 1.98 & 1.15 & 0.95 & 0.81 & 5 & 31 & 30 & 3 & 1 & 3 & 20 & 21 & 1 & 1 & 1.4 & 6.0 \\
\hline run103 & 12 & 29 & 0.09 & 0.17 & 2.99 & 1.96 & 0.94 & 0.80 & 2 & 41 & 34 & 1 & 2 & 1 & 27 & 22 & 0 & 0 & 1.1 & 8.1 \\
\hline run104 & 12 & 36 & 0.09 & 0.16 & 1.94 & 0.84 & 1.11 & 1.36 & 4 & 65 & 33 & 8 & 0 & 3 & 42 & 23 & 4 & 0 & 1.2 & 9.4 \\
\hline run105 & 12 & 15 & 0.18 & 0.31 & 0.67 & 0.58 & 1.15 & 0.89 & 1 & 24 & 44 & 6 & 4 & 0 & 17 & 31 & 3 & 2 & 1.4 & 5.9 \\
\hline run106 & 12 & 23 & 0.18 & 0.30 & 1.14 & 0.79 & 1.13 & 1.03 & 1 & 41 & 65 & 7 & 1 & 0 & 27 & 41 & 6 & 1 & 1.3 & 7.3 \\
\hline run107 & 12 & 30 & 0.18 & 0.29 & 1.07 & 0.94 & 1.27 & 1.02 & 2 & 61 & 74 & 8 & 2 & 1 & 43 & 62 & 2 & 2 & 1.3 & 7.1 \\
\hline run108 & 12 & 38 & 0.18 & 0.27 & 0.93 & 3.95 & 1.40 & 0.50 & 3 & 70 & 66 & 7 & 5 & 2 & 43 & 52 & 5 & 0 & 1.7 & 10.1 \\
\hline run109* & 12 & 15 & 0.27 & 0.41 & 0.46 & 0.50 & 1.30 & 1.09 & 0 & 21 & 67 & 6 & 1 & 0 & 12 & 42 & 1 & 0 & 1.4 & 9.3 \\
\hline run110 & 12 & 23 & 0.27 & 0.40 & 0.67 & 73.41 & 1.34 & 0.11 & 0 & 44 & 99 & 3 & 5 & 0 & 30 & 67 & 1 & 1 & 1.4 & 7.3 \\
\hline run111 & 12 & 32 & 0.27 & 0.39 & 0.65 & 3.93 & 1.47 & 0.43 & 1 & 70 & 128 & 10 & 5 & 1 & 42 & 85 & 3 & 2 & 1.4 & 6.9 \\
\hline run112 & 12 & 40 & 0.27 & 0.36 & 0.65 & 2.35 & 1.58 & 0.71 & 5 & 60 & 112 & 14 & 4 & 2 & 38 & 71 & 4 & 3 & 1.7 & 7.9 \\
\hline run113* & 12 & 13 & 0.05 & 0.10 & 1.93 & 1.36 & 0.80 & 0.54 & 1 & 16 & 9 & 0 & 0 & 1 & 7 & 6 & 0 & 0 & 1.6 & 5.7 \\
\hline run114* & 12 & 21 & 0.05 & 0.10 & 4.39 & 1.51 & 0.71 & 0.66 & 4 & 24 & 12 & 0 & 0 & 0 & 16 & 7 & 0 & 0 & 1.5 & 6.7 \\
\hline run115* & 12 & 28 & 0.04 & 0.09 & 7.53 & 5.29 & 0.69 & 0.47 & 3 & 34 & 17 & 0 & 0 & 2 & 16 & 11 & 0 & 0 & 1.6 & 4.7 \\
\hline run116* & 12 & 35 & 0.04 & 0.09 & 9.44 & 4.24 & 0.70 & 0.56 & 6 & 53 & 16 & 1 & 1 & 2 & 35 & 12 & 0 & 0 & 1.7 & 6.6 \\
\hline run117* & 12 & 14 & 0.09 & 0.17 & 0.97 & 0.34 & 1.00 & 1.07 & 2 & 18 & 10 & 4 & 2 & 0 & 11 & 6 & 0 & 0 & 1.5 & 4.1 \\
\hline run118* & 12 & 21 & 0.09 & 0.17 & 2.46 & 8.78 & 0.87 & 0.21 & 3 & 32 & 43 & 1 & 4 & 2 & 21 & 31 & 0 & 3 & 1.6 & 6.3 \\
\hline run119* & 12 & 29 & 0.09 & 0.16 & 4.32 & 1.06 & 0.84 & 1.00 & 4 & 50 & 41 & 1 & 1 & 0 & 25 & 28 & 0 & 0 & 1.5 & 5.9 \\
\hline run120* & 12 & 36 & 0.09 & 0.16 & 3.98 & 1.15 & 0.92 & 1.06 & 4 & 66 & 61 & 0 & 4 & 2 & 43 & 38 & 0 & 3 & 1.5 & 8.6 \\
\hline run121* & 12 & 14 & 0.18 & 0.30 & 0.70 & 2.15 & 1.15 & 0.39 & 0 & 23 & 37 & 5 & 0 & 0 & 13 & 24 & 2 & 0 & 1.6 & 7.3 \\
\hline run122* & 12 & 22 & 0.18 & 0.31 & 2.31 & 12.09 & 0.94 & 0.23 & 1 & 52 & 79 & 1 & 1 & 1 & 34 & 52 & 1 & 0 & 1.6 & 6.1 \\
\hline run123* & 12 & 30 & 0.18 & 0.29 & 3.39 & 3.21 & 0.90 & 0.49 & 3 & 53 & 102 & 0 & 2 & 1 & 32 & 60 & 0 & 1 & 1.4 & 7.6 \\
\hline run124* & 12 & 38 & 0.18 & 0.29 & 4.83 & 2.56 & 0.91 & 0.73 & 5 & 90 & 146 & 4 & 7 & 1 & 49 & 97 & 1 & 4 & 1.7 & 6.4 \\
\hline run125* & 12 & 15 & 0.27 & 0.42 & 0.95 & 11.82 & 1.04 & 0.15 & 1 & 22 & 47 & 2 & 0 & 0 & 10 & 26 & 0 & 0 & 1.4 & 6.6 \\
\hline run126* & 12 & 23 & 0.27 & 0.41 & 1.84 & 36.82 & 1.00 & 0.08 & 0 & 44 & 124 & 2 & 1 & 0 & 27 & 82 & 1 & 0 & 1.3 & 7.1 \\
\hline run127 & 12 & 31 & 0.27 & 0.41 & 3.13 & 12.32 & 0.95 & 0.27 & 4 & 51 & 160 & 1 & 3 & 1 & 23 & 109 & 0 & 1 & 1.4 & 7.5 \\
\hline run128 & 12 & 39 & 0.27 & 0.39 & 2.61 & 1.77 & 1.07 & 0.89 & 2 & 79 & 204 & 3 & 5 & 0 & 45 & 119 & 2 & 0 & 1.6 & 10.9 \\
\hline
\end{tabular}

Note. $-t_{\mathrm{cl}}$ denotes the cluster age in Gyr, $M$ denotes the total cluster mass at $t_{\mathrm{cl}}$ in $10^{4} M_{\odot}, f_{b}$ denotes the overall binary fraction in the cluster, $f_{b, c}$ denotes the binary fraction in the core, $\rho_{c}$ denotes the core density in $10^{4} M_{\odot} \mathrm{pc}^{-3}$, and $r_{c}$ denotes the core radius in pc. The corresponding "observed" values are given as $r_{c, \text { obs }}$ and $\rho_{c, \text { obs }}$. $N_{\mathrm{BSS}}$ and $N_{\mathrm{BSS}, \mathrm{c}}$ denote the numbers of BSSs in the whole cluster, and within $r_{c}$, respectively. The time since formation for the BSSs is denoted by $t_{\text {age }}$ in Gyr. Coll, MTB, and SE denote BSSs formed via stellar collisions, stable mass-transfer in a binary, and binary stellar evolution driven mergers, respectively. s-s, b-s, and $\mathrm{b}-\mathrm{b}$ denote collisions via single-single, binary-single, and binary-binary encounters, respectively. Med, and Max denote the median $t_{\mathrm{age}}$ and the maximum $t_{\text {age }}$ for each model. Models that are in the quasi-steady binary-burning stage (equivalent to post core-collapsed; Chatteriee et al. 2013) are marked by “*”. 